\title{
Positivity of the T-system cluster algebra
}

\author{
Philippe Di Francesco \\ Insitut de Physique Théorique du Commissariat à l'Energie Atomique, \\ Unité de recherche associeée du CNRS, CEA Saclay/IPhT/Bat 774, \\ F-91191 Gif sur Yvette Cedex, France \\ philippe.di-francesco@cea.fr \\ Rinat Kedem \\ Department of Mathematics, University of Illinois, Urbana, IL 61821, USA \\ rinat@illinois.edu \\ Submitted: Sep 10, 2009; Accepted: Nov 12, 2009; Published: Nov 24, 2009 \\ Mathematics Subject Classification: 05C88
}

\begin{abstract}
We give the path model solution for the cluster algebra variables of the $T$ system of type $A_{r}$ with generic boundary conditions. The solutions are partition functions of (strongly) non-intersecting paths on weighted graphs. The graphs are the same as those constructed for the $Q$-system in our earlier work, and depend on the seed or initial data in terms of which the solutions are given. The weights are "time-dependent" where "time" is the extra parameter which distinguishes the $T$-system from the $Q$-system, usually identified as the spectral parameter in the context of representation theory. The path model is alternatively described on a graph with non-commutative weights, and cluster mutations are interpreted as noncommutative continued fraction rearrangements. As a consequence, the solution is a positive Laurent polynomial of the seed data.
\end{abstract}

\section{Introduction}

In this paper we study solutions of the $T$-system associated to the Lie algebras $A_{r}$, which we write in the following form:

$$
T_{\alpha, j, k+1} T_{\alpha, j, k-1}=T_{\alpha, j+1, k} T_{\alpha, j-1, k}+T_{\alpha+1, j, k} T_{\alpha-1, j, k},
$$

where $j, k \in \mathbb{Z}, \alpha \in I_{r}=\{1, \ldots, r\}$, and with boundary conditions

$$
T_{0, j, k}=T_{r+1, j, k}=1, \quad j, k \in \mathbb{Z} .
$$


We consider these equations to be discrete evolution equations for the commutative variables $\left\{T_{\alpha, j, k}\right\}$ in the direction of the discrete variable $k$.

Originally, this relation appeared as the fusion relation for the commuting transfer matrices of the generalized Heisenberg model [1, 18] associated with a simply-laced Lie algebra $\mathfrak{g}$, where it is written in the form

$$
\bar{T}_{\alpha, j, k+1} \bar{T}_{\alpha, j, k-1}=\bar{T}_{\alpha, j+1, k} \bar{T}_{\alpha, j-1, k}-\prod_{\beta \neq \alpha} \bar{T}_{\beta, j, k}^{-C_{\beta, \alpha}}
$$

with appropriate boundary conditions. The matrix $C$ is the symmetric Cartan matrix of one of the Lie algebra of type $A D E$. Our relation (1.1) is obtained by a rescaling of the variables $\bar{T}_{\alpha, j, k}$ and specializing to the Cartan matrix of type $A_{r}$.

With special initial condition at $k=0$, it has been proved that the solutions to (1.3) are the $q$-characters [11] of the Kirillov-Reshetikhin modules of the quantum affine Lie algebra $U_{q}\left(\widehat{s}_{r+1}\right)[20]$.

The $T$-system also appears in several other contexts. Of particular relevance here is the fact [19] that the system is a discrete integrable equation, the discrete Hirota equation. It is therefore to be expected that the system has a complete set of integrals of motion, and that it is exactly solvable. This equation also appears in a related combinatorial context, as the octahedron equation, which was studied by [17, 23].

In this paper, we do not impose any special boundary conditions, but express the general solution of the $T$-system in terms of arbitrary initial conditions. For example, initial conditions can be chosen by specifying the values of the parameters $T_{\alpha, j, k}$ at $k=0$ and $k=1$, or a more exotic boundary can be specified. To solve the system, we use a path model which is a simple generalization of the path model we constructed for the solutions of the $Q$ - system of type $A_{r}[6,7]$.

In our previous work, we constructed a set of path models, and proved that the solutions of the $Q$-system of type $A_{r}[16]$,

$$
Q_{\alpha, k+1} Q_{\alpha, k-1}=Q_{\alpha, k}^{2}+Q_{\alpha-1, k} Q_{\alpha+1, k}, \quad Q_{0, k}=Q_{r+1, k}=1 ; k \in \mathbb{Z}, \alpha \in I_{r},
$$

are the generating functions for paths on a positively weighted graph, where the weights are a function of the initial conditions.

With special initial conditions at $k=0$ and $k=1$ (together with a rescaling as in (1.3) which restores the minus sign in the second term on the right hand side of the $Q$-system), the solutions are the characters the finite- dimensional, irreducible modules of $A_{r}$ with highest weights which are multiples of one of the fundamental weights.

Note that this $Q$-system is obtained by "forgetting" the spectral parameter $j$ in Equation (1.1). Thus the $T$-system can be regarded as an affinization or $q$-deformation of the $Q$-system, and the path model we present here is therefore a deformation of the path model for the $Q$-system.

Without fixing any special initial conditions, it was shown in [14] that the solutions of the $Q$-system are cluster variables in a cluster algebra [9]. We showed in [5] that all $Q$-systems, corresponding to any simple Lie algebra, can be formulated as cluster 
algebras. The fundamental, built-in property of cluster algebras, is the fact that all cluster variables may be expressed as Laurent polynomials of the variables in any other cluster. More surprising but very robust is the observed positivity of the coefficients of these polynomials, leading to the general positivity conjecture of [9], proved only in a few cases so far (see e.g. [22] for the case of rank two cluster algebras and [4] for the case of acyclic cluster algebras). The solution of the $Q$-system in terms of the statistical model allowed us to prove the positivity conjecture of [9] for these cluster variables. In fact, as we showed in [7], the solutions are related to the totally positive matrices of [10] corresponding to pairs of coxeter elements.

Similarly, we showed in [5] that a large class of equations which we call generalized bipartite $T$-systems can be formulated as cluster algebras. Equation (1.1) is perhaps the simplest example of such a system. Our aim in the present paper, is to prove the positivity conjecture for the cluster variables of the $T$-system of type $A_{r}$.

Motivated by our statistical model introduced in [6], we introduce a path model which provides us with the solution to the $T$-system, in terms of a set of initial conditions, as the partition function of a path model with time-dependent (or non-commutative) weights. Here, we refer to the variable normally identified as the spectral parameter as the time parameter, as it is a natural interpretation from the point of view of paths.

This paper is organized as follows. In Section 2, we review the necessary definition of a cluster algebra. We recall our formulation [5] of $T$-systems as cluster algebras. We describe the conserved quantities of the $T$-system in terms of discrete Wronskian determinants in Section 3. We define a generalized notion of hard particle models on a graph in Section 4 and identify the conserved quantities as hard particle partition functions on a specific graph. In Section 5, we use our conserved quantities to write the solutions of the $T$-system as the partition functions of paths on a weighted graph. The weight of a step in a path depends on the order in which the steps are taken, that is, the weights are time-dependent. The solutions are written as functions of the fundamental initial data, and the graph is the same as the one used in the $Q$-system solution. Positivity of the $T$-system solutions in terms of the fundamental seed variables follows from this formulation.

To prove the positivity in terms of other seeds, we give a formulation of our model in terms of non-commutative weights in Section 6. We are then able to describe the solutions of the $T$-system as a function of other seed data as partition functions on new graphs with weights which depend on the mutated seeds. The key to the construction is an operator version of the fraction rearrangement lemmas used in [5]. These rearrangements are equivalent to mutations in the case of the $Q$-system. Here, they are equivalent to compound mutations. We are thus able to write the $T$-system solution explicitly in terms of its initial data, for a subset of cluster seeds.

This paper should be considered as a (special case of) non-commutative generalization of our work on the solutions of $Q$-system [6,7], by viewing the (commuting) cluster variables as eigenvalues of (non-commuting) operators. In particular, the graphs on which we build our path models are the same as for the $Q$-system, and the only difference is that we must now keep track of the time-dependence hence of the chronological order of the path steps: this is achieved by introducing non-commutative operator weights. The 
various key properties, such as the rearrangement lemmas for continued fractions and the generalization of the Lindström-Gessel-Viennot theorem for strongly non-intersecting paths, all have straightforward non-commutative counterparts which are used here.

Acknowledgements: P.D.F.'s research is supported in part by the ANR Grant GranMa, the ENIGMA research training network MRTN-CT-2004-5652, and the ESF program MISGAM. R.K.'s research is supported by NSF grant DMS-0802511. R.K. thanks IPhT at CEA/ Saclay for their kind hospitality. We also acknowledge the hospitality of the Mathematisches Forschungsinstituts Oberwolfach (RIP program), where this paper was completed.

\section{$2 \quad T$-systems as cluster algebras}

\section{$2.1 \quad$ Cluster algebras}

We use the following definition of a cluster algebra [9, 24], slightly specialized to suit our needs in this paper.

Let $S \subset \widetilde{S}$ be two discrete sets (possibly infinite) and consider the field $\mathcal{F}$ of rational functions over $\mathbb{Q}$ in a set of independent variables indexed by $\widetilde{S}$.

We define a seed in $\mathcal{F}$ to be a pair $(\widetilde{\mathbf{x}}, \widetilde{B})$, where $\widetilde{\mathbf{x}}=\left\{x_{m}: m \in \widetilde{S}\right\}$ is a set of commuting variables, and $\widetilde{B}$ is an integer matrix, with rows indexed by $\widetilde{S}$ and columns indexed by $S$. The matrix $B$, which is the square submatrix of $\widetilde{B}$ made up of the rows of $\widetilde{B}$ indexed by $S$, is skew symmetric.

The cluster of the seed $(\widetilde{x}, \widetilde{B})$ is the set of variables $\left\{x_{m}: m \in S\right\}$, and the coefficients are the set of variables $\left\{x_{m}: m \in \widetilde{S} \backslash S\right\}$.

Next, we define a seed mutation. For any $m \in S$, a mutation in the direction $m$, $\mu_{m}:(\widetilde{x}, \widetilde{B}) \mapsto\left(\widetilde{x}^{\prime}, \widetilde{B^{\prime}}\right)$, is a discrete evolution of the seed. Explicitly,

- The mutation $\mu_{m}$ leaves $x_{n}$ with $n \neq m$ invariant, and updates the variable $x_{m}$ only, via the exchange relation

$$
x_{m}^{\prime}=x_{m}^{-1}\left(\prod_{n \in \widetilde{S}} x_{n}^{\left[\widetilde{B}_{n, m}\right]_{+}}+\prod_{n \in \widetilde{S}} x_{n}^{\left[-\widetilde{B}_{n, m}\right]_{+}}\right)
$$

where $[n]_{+}=\max (n, 0)$.

- The exchange matrix $\widetilde{B}^{\prime}$ has entries

$$
\widetilde{B}_{i, j}^{\prime}= \begin{cases}-\widetilde{B}_{i, j} & \text { if } i=m \text { or } j=m \\ \left.\widetilde{B}_{i, j}+\operatorname{sign}\left(\widetilde{B}_{i, m}\right)\left[\widetilde{B}_{i, m} \widetilde{B}_{m, j}\right]_{+}\right) & \text {otherwise. }\end{cases}
$$

Note that we only define mutations for the set $S$, and not for the coefficient set $\widetilde{S} \backslash S$. That is, coefficients do not evolve. 
Fix a seed $(\widetilde{\mathbf{x}}, \widetilde{B})$ and consider the orbit $X \subset \mathcal{F}$ of the cluster variables under all combinations of the mutations $\mu_{m}, m \in S$. The cluster algebra is the $\mathbb{Z}\left[\mathbf{c}^{ \pm 1}\right]$ - subalgebra of $\mathcal{F}$ generated by $\mathcal{X}$, where $\mathbf{c}$ is the common coefficient set of the orbit of the seed.

Remark 2.1. The particular system which we solve in this paper does not require us to have a coefficient set, that is, we can set $S=\widetilde{S}$. However, to make more direct contact with representation theory, it is desirable to have the coefficient set be enumerated by the roots of the Lie algebra. In this context, we need to set the values of the coefficients to the special points -1 .

Cluster algebras can be considered to be discrete dynamical systems, which is the point of view we adopt in this paper.

\subsection{Bipartite $T$-systems as cluster algebras}

In this section we review some of the definitions of Appendix B of [5], where generalized bipartite $T$-systems were shown to have a cluster algebra structure.

Definition 2.2. A generalized bipartite $T$-system is a recursion relation for the commuting, invertible variables $\left\{T_{\alpha, j ; k}\right\}$, where $\alpha \in I_{r}$ and $j, k \in \mathbb{Z}$, of the form

$$
T_{\alpha, j ; k+1} T_{\alpha, j ; k-1}=T_{\alpha, j+1 ; k} T_{\alpha, j-1 ; k}+q_{\alpha} \prod_{j^{\prime}} \prod_{\alpha^{\prime}}\left(T_{\alpha^{\prime}, j^{\prime} ; k}\right)^{A_{\alpha^{\prime}, \alpha}^{j^{\prime}, j}}
$$

where $A$ is an incidence matrix, that is, a symmetric matrix with positive integer entries.

The matrix $A$ is generally of infinite size, unless special boundary conditions are imposed on the system which truncate the range of the variables $j$. We do not impose such boundary conditions in this paper, although they are clearly of interest [13, 21]. The symmetry of $A$ is required for the bipartite property to hold (see below). T-systems which are not bipartite can also be defined, and in that case, the matrix $A$ is not symmetric.

Example 2.3. The first example of such a $T$ system is the one described in (1.3). In that case, we take the matrix $A$ to be as follows:

$$
A_{\alpha, \beta}^{j, j^{\prime}}=\mathcal{J}_{\alpha, \beta} \delta_{j, j^{\prime}}
$$

where $\mathcal{J}_{\alpha, \beta}=C-2 I$ is the incidence matrix of the Dynkin diagram associated with a simply-laced Lie algebra $\mathfrak{g}$. The coefficients $q_{\alpha}$ are all set to be -1 . However, it is always possible to renormalize the variables so that $q_{\alpha}=1$ in these cases [14], and we use this approach here.

In particular, if $\mathfrak{g}=A_{r},(\mathcal{J})_{\alpha, \beta}=\delta_{\alpha, \beta+1}+\delta_{\alpha, \beta-1}$. This is the case we solve in this paper.

We note that another example of generalized $T$-systems appeared in the context of preprojective algebras and the categorification program of [12]. The explicit connection was made in [5], Example 4.4. 
Finally, define the (possibly infinite) matrix $P$ with entries

$$
P_{\alpha, \beta}^{j, l}=\delta_{\alpha, \beta}\left(\delta_{i, j+1}+\delta_{i, j-1}\right) .
$$

Then we can rewrite (2.3) as

$$
T_{\alpha, j ; k+1} T_{\alpha, j ; k-1}=\prod_{\alpha, j} T_{\beta, j^{\prime}}^{P_{\beta}^{j^{\prime}, j}}+q_{\alpha} \prod_{j^{\prime}} \prod_{\alpha^{\prime}}\left(T_{\alpha^{\prime}, j^{\prime} ; k}\right)^{A_{\alpha^{\prime}, \alpha}^{j^{\prime}, j}} .
$$

In the systems considered in [5], we allowed the matrix $P$ to be a matrix with positive integer entries, such that it commutes with the matrix $A$, together with another condition on the sum of its entries (see Lemma 2.5 below). Such a system is also a generalized bipartite $T$ - system.

\subsection{Cluster algebra structure}

We recall the formulation found in Appendix B of [5] of the cluster algebra associated with generalized (bipartite) $T$-systems.

In the notations of Section 2, let $S=\left(I_{r} \sqcup \bar{I}_{r}\right) \times Z$, and $\widetilde{S}=S \sqcup I_{r}^{\prime}$. Each set $I_{r}, \bar{I}_{r}$ and $I_{r}^{\prime}$ is just the set with $r$ elements. For convenience, if $\alpha \in I_{r}$, then by $\bar{\alpha}$ we mean the $\alpha$ th element of $\bar{I}_{r}$, etc.

We define the fundamental seed $(\widetilde{\mathbf{x}}, \widetilde{B})_{0}$ as follows. The variables $\widetilde{\mathbf{x}}_{0}$ are

$$
\begin{aligned}
& x_{\alpha, j}=T_{\alpha, j ; 0}, \quad\left(\alpha \in I_{r}, j \in \mathbb{Z}\right) ; \\
& x_{\bar{\alpha}, j}=T_{\alpha, j ; 1},\left(\bar{\alpha} \in \bar{I}_{r}, j \in \mathbb{Z}\right) ; \\
& x_{\alpha^{\prime}}=q_{\alpha}, \alpha^{\prime} \in I_{r}^{\prime} ;
\end{aligned}
$$

The elements of the set $\left\{x_{\alpha, j}\right\} \sqcup\left\{x_{\bar{\alpha}, j}\right\}$ are the cluster variables and $\left\{x_{\alpha^{\prime}}\right\}$ are the coefficients. The exchange matrix of the fundamental seed is defined as follows:

$$
\begin{aligned}
& B_{\alpha, j ; \beta, l}=0, \quad\left(\alpha, \beta \in I_{r}, j, l \in \mathbb{Z}\right), \quad B_{\bar{\alpha}, j ; \bar{\beta}, l}=0, \quad\left(\bar{\alpha}, \bar{\beta} \in \bar{I}_{r}, j, l \in \mathbb{Z}\right), \\
& B_{\alpha, j ; \bar{\beta}, l}=-P_{\alpha, \beta}^{j, l}+A_{\alpha, \beta}^{j, l}=-B_{\overline{\bar{\beta}}, l ; \alpha, j} \\
& \widetilde{B}_{\alpha^{\prime} ; \beta, j}=-\widetilde{B}_{\alpha^{\prime}, \bar{\beta}, j}=-\delta_{\alpha, \beta} .
\end{aligned}
$$

The last equation above denotes the entries of the extended $B$-matrix, corresponding to the coefficients, which do not mutate. The matrices $A, P$ are those of equation (2.6) for the generalized $T$-system.

Example 2.4. In the case of the $A_{r}$ system (1.1), we have the matrix $A$ as in (2.4), $P$ as in (2.5) and $q_{\alpha}=1$. In that case we do not need to include the coefficients $q_{\alpha}$, and the matrix $\widetilde{B}$ is equal to the matrix $B$. To recover the original $T$-system (1.3), we take $q_{\alpha}=-1$. 
It is clear that each of the mutations $\mu_{\alpha, j}$ and $\mu_{\bar{\alpha}, j}$ exchanges one of the cluster variables in $\widetilde{\mathbf{x}}_{0}$ via one of the $T$-system equation relations (2.6). The mutation $\mu_{\alpha, j}$ acts on $\widetilde{\mathbf{x}}_{0}$ as one of the $T$-system evolutions (2.6), where we specialize to $k=1: \mu_{\alpha, j}\left(T_{\alpha, j ; 0}\right)=T_{\alpha, j ; 2}$. Similarly, $\mu_{\bar{\alpha}, j} T_{\alpha, j ; 1}=T_{\alpha, j ;-1}$ is a $T$-system equation specialized to $k=0$.

Quite generally, if $B_{a, b}=0$ then $\mu_{a} \circ \mu_{b}=\mu_{b} \circ \mu_{a}$. Since $B_{\alpha, j ; \beta, l}=0$ for all $\alpha, \beta \in I_{r}$ and $j, l \in \mathbb{Z}$, when acting on the initial seed $(\widetilde{\mathbf{x}}, \widetilde{B})_{0}$, the mutations $\mu_{\alpha, m}$ commute with each other for all $\alpha, m$. Similarly the mutations $\mu_{\bar{\alpha}, m}$ also commute among themselves.

Therefore we can define the compound mutations

$$
\mu:=\prod_{\alpha, m} \mu_{\alpha, m}, \quad \bar{\mu}:=\prod_{\bar{\alpha}, m} \mu_{\bar{\alpha}, m}
$$

which act on $(\widetilde{\mathbf{x}}, \widetilde{B})_{0}$. More generally, we can define $(\widetilde{\mathbf{x}}, \widetilde{B})_{2 k}$ to be the seed with $x_{\alpha, j}=$ $T_{\alpha, j ; 2 k}, x_{\bar{\alpha}, j}=T_{\alpha, j ; 2 k+1}$ and $\widetilde{B}_{2 k}=\widetilde{B}$. Define $(\widetilde{\mathbf{x}}, \widetilde{B})_{2 k+1}$ to be the seed with $x_{\alpha, j}=$ $T_{\alpha, j ; 2 k+2}, x_{\bar{\alpha}, j}=T_{\alpha, j ; 2 k}$ and $\widetilde{B}_{2 k+1}=-\widetilde{B}$. Then it is clear that $\mu\left(\widetilde{\mathbf{x}}_{2 k}\right)=\widetilde{\mathbf{x}}_{2 k+1}$ : Each mutation $\mu_{\alpha, j}$ mutates the variable $T_{\alpha, j ; 2 k}$ into the variable $T_{\alpha, j ; 2 k+2}$. Similarly, it is easy to check that $\bar{\mu}\left(\widetilde{\mathbf{x}}_{2 k}\right)=\widetilde{\mathbf{x}}_{2 k-1}, \mu\left(\widetilde{\mathbf{x}}_{2 k+1}\right)=\widetilde{\mathbf{x}}_{2 k}$ and $\bar{\mu}\left(\widetilde{\mathbf{x}}_{2 k+1}\right)=\widetilde{\mathbf{x}}_{2 k+2}$.

The following statement is Lemma 4.6 of [5]:

Lemma 2.5. Assume that the matrix $A$ commutes with the matrix $P$, and that

$$
\sum_{k} P_{\alpha, \beta}^{k j}=2 \delta_{\alpha, \beta}
$$

for any $j$. Then the cluster algebra $X$ which includes the seed $(\widetilde{\mathbf{x}}, \widetilde{B})_{0}$ as in $(2.7),(2.8)$ includes all the solutions of the T-system (2.6). All the T-system relations are exchange relations in this cluster algebra.

To prove this Lemma, we need

\section{Lemma 2.6.}

$$
\mu\left((\widetilde{\mathbf{x}}, \widetilde{B})_{2 k}\right)=(\widetilde{\mathbf{x}}, \widetilde{B})_{2 k+1}, \quad \bar{\mu}\left((\widetilde{\mathbf{x}}, \widetilde{B})_{2 k}\right)=(\widetilde{\mathbf{x}}, \widetilde{B})_{2 k-1}
$$

Proof. In light of the preceding discussion, all that needs to be proved is that $\mu(\widetilde{B})=$ $\bar{\mu}(\widetilde{B})=-\widetilde{B}$. Let $\widetilde{B}^{\prime}=\mu(\widetilde{B})$. Then, since $B_{\alpha, j ; \beta, k}=0$, we have

- $\mu_{\alpha, i}\left(B_{\beta, j ; \gamma, k}\right)=\operatorname{sign}\left(B_{\beta, j ; \alpha, i}\right)\left[B_{\beta, j ; \alpha, i} B_{\alpha, i ; \gamma, k}\right]_{+}=0$;

- $\mu_{\alpha, i}\left(B_{\bar{\beta}, j ; \gamma, k}\right)=-B_{\bar{\beta}, j ; \gamma, k}$ if $(\alpha, i)=(\gamma, k)$, and is otherwise unchanged, since if $(\alpha, i) \neq(\gamma, k)$,

$$
\mu_{\alpha, i}\left(B_{\bar{\beta}, j ; \gamma, k}\right)=B_{\bar{\beta}, j ; \gamma, k}+\operatorname{sign}\left(B_{\bar{\beta}, j ; \alpha, i}\right)\left[B_{\bar{\beta}, j ; \alpha, i} B_{\alpha, i ; \gamma, k}\right]_{+}=B_{\bar{\beta}, j ; \gamma, k} .
$$

Similarly, $\mu_{\alpha, i}\left(B_{\beta, j ; \bar{\gamma}, k}\right)=-B_{\beta, j ; \bar{\gamma}, k}$. 


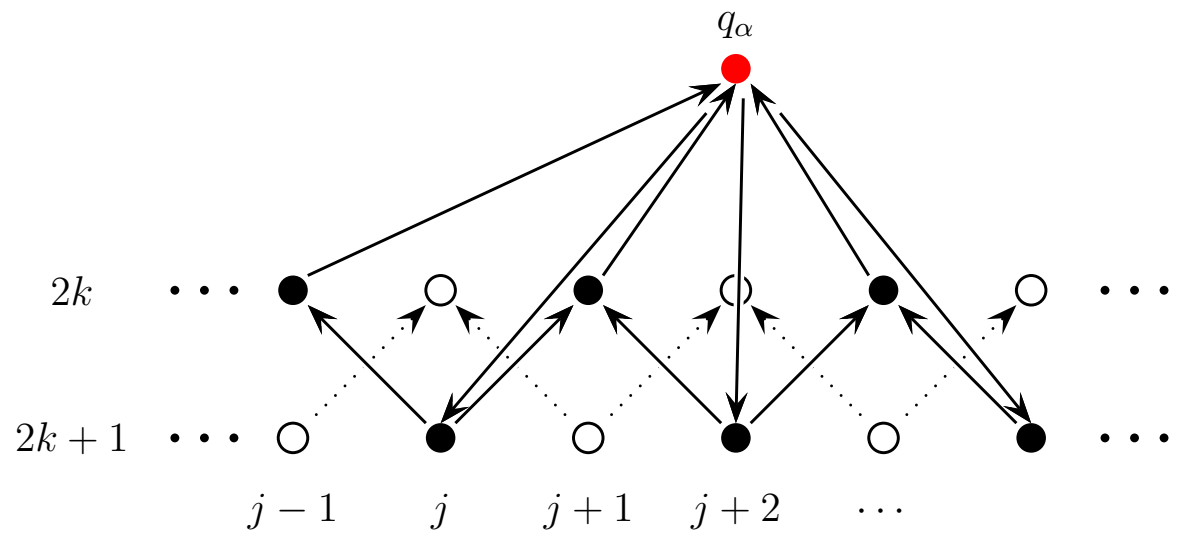

Figure 2.1: A slice of the quiver graph of $\widetilde{B}$, corresponding to constant $\alpha$. The nodes in the strip are labeled by $(j, k)$ of $T_{\alpha, j ; k}$. The two subgraphs with even and odd $j+k$ decouple in this slice, so we illustrate the only the connectivity of nodes of the same parity to node $q_{\alpha}$. The mutation $\mu$ reverses all arrows connected to $q_{\alpha}$.

- Recall the restriction that $[P, A]=0$. Then

$$
\mu\left(B_{\bar{\beta}, j ; \bar{\gamma}, k}\right)=\sum_{\alpha, i} \operatorname{sign}\left(B_{\bar{\beta}, j ; \alpha, i}\right)\left[B_{\bar{\beta}, j ; \alpha, i} B_{\alpha, i ; \bar{\gamma}, k}\right]_{+}=(P A-A P)_{\beta, \gamma}^{j, k}=0 .
$$

- We have $\mu_{\alpha, i}\left(B_{\beta^{\prime} ; \gamma, k}\right)=-B_{\beta^{\prime} ; \gamma, k}$, and otherwise, if $(\alpha, i) \neq(\gamma, k)$ then $\mu_{\alpha, i}$

$$
\mu_{\alpha, i}\left(\widetilde{B}_{\beta^{\prime} ; \gamma, k}\right)=\widetilde{B}_{\beta^{\prime} ; \gamma, k}+\sum_{\alpha, i} \operatorname{sign}\left(\widetilde{B}_{\beta^{\prime} ; \alpha, i}\right)\left[\widetilde{B}_{\beta^{\prime} ; \alpha, i} B_{\alpha, i ; \gamma, k}\right]=\delta_{\beta, \gamma},
$$

so that $\mu\left(\widetilde{B}_{\beta^{\prime} ; \gamma, k}\right)=-\widetilde{B}_{\beta^{\prime} ; \gamma, k}$.

- Finally, using the restriction (2.9) on the summation of elements of $P$,

$$
\mu\left(\widetilde{B}_{\beta^{\prime} ; \bar{\gamma}, k}\right)=\delta_{\beta, \gamma}+\sum_{\alpha, i} \operatorname{sign}\left(\widetilde{B}_{\beta^{\prime} ; \alpha, i}\right)\left[\widetilde{B}_{\beta^{\prime} ; \alpha, i} B_{\alpha, i ; \bar{\gamma}, k}\right]_{+}=\delta_{\beta, \gamma}-\sum_{\alpha} \delta_{\alpha, \beta} \sum_{i} P_{\alpha, \gamma}^{i, k}=-\delta_{\beta, \gamma} .
$$

In the quiver graph corresponding to $\widetilde{B}$, the last two statements are about how nodes $x_{\alpha, j}=T_{\alpha, 2 k}$ and $x_{\bar{\alpha}, j}=T_{\alpha, j ; 2 k+1}$ are connected to node $x_{\beta^{\prime}}=q_{\beta}$. If $\alpha \neq \beta$, they are not connected, and if $\alpha=\beta$, the connectivity is illustrated in Figure 2.3 and the mutations in Figure 2.3.

We have shown that $\mu(\widetilde{B})=-\widetilde{B}$. The proof that $\bar{\mu}(\widetilde{B})=-\widetilde{B}$ is similar.

Thus, we have shown that all the variables $T_{\alpha, j ; k}$ appear in the cluster algebra, in fact, within a bipartite graph composed of the nodes reached from $(\widetilde{\mathbf{x}}, \widetilde{B})_{0}$ via combinations of the compound mutations $\mu$ and $\bar{\mu}$ only. 


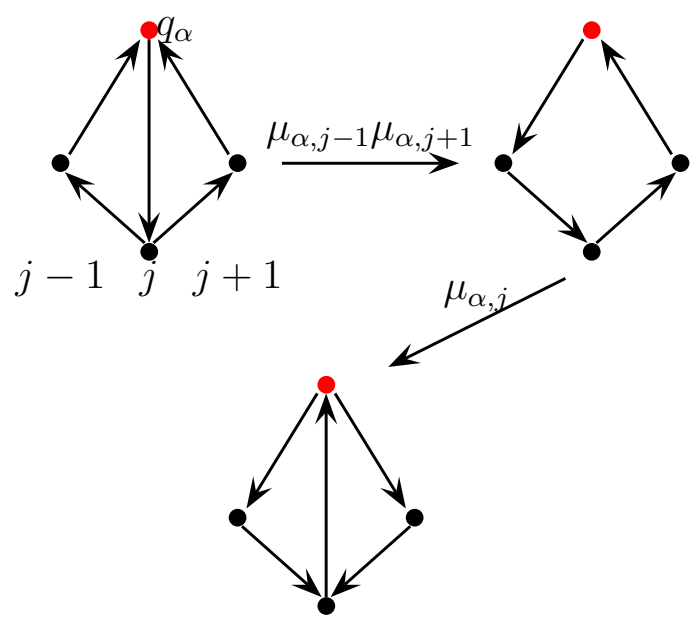

Figure 2.2: The local action of the mutation $\mu$ on a section of the quiver graph. The compound mutation reverses all arrows connected to $q_{\alpha}$.

In this paper, we study the solutions of the $T$-system of type $A_{r}$ in terms of the fundamental seed cluster $\widetilde{\mathbf{x}}_{0}$. The result will be an explicit interpretation of the solutions as partition functions of paths on a graph whose weights which are positive monomials in the variables $\widetilde{\mathbf{x}}_{0}$. This will imply the positivity property [9] for the cluster variables $T_{\alpha, j ; k}$ : They can be expressed as Laurent polynomials with non-negative coefficients in terms of the initial data.

\section{Basic properties of the $T$-system}

From here on, we specialize the discussion to the $T$-system (1.1). Equation (1.1) is a three-term recursion in the index $k$, which allows us to determine all the $\left\{T_{\alpha, j, k+1}\right\}_{\alpha \in I_{r}, j \in \mathbb{Z}}$

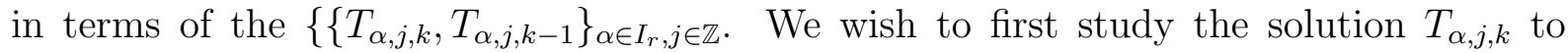

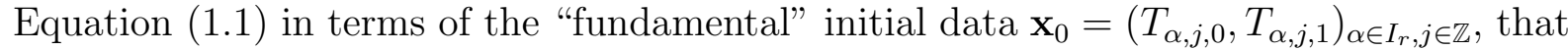
is, $\mathbf{x}_{0}$. The techniques used in this section are a straightforward generalization of the methods used for the $Q$-system in [6]. We therefore present the proofs of the theorems in the Appendix, as they use standard techniques in the theory of determinants.

\subsection{Discrete Wronskians and conserved quantities}

We can express the subset of variables $\left\{T_{\alpha, j, k}: j, k \in \mathbb{Z}, \alpha>1\right\}$ as polynomials of the variables in the set $\left\{T_{1, j, k}: j, k \in \mathbb{Z}\right\}$, cf $[18]$ :

\section{Theorem 3.1.}

$$
T_{\alpha, j, k}=\operatorname{det}_{1 \leqslant a, b \leqslant \alpha}\left(T_{1, j-a+b, k+a+b-\alpha-1}\right), \quad \alpha \in I_{r}, j, k \in \mathbb{Z}
$$


The proof of this theorem uses the standard Plücker relations, and is similar to the case of the $Q$-system. We therefore present the details of the proof in the Appendix, Section A.2.

If we consider $\alpha=r+1$ in Equation (3.1), since $T_{r+1, j ; k}=1$, we have the polynomial relation among the variables $\left\{T_{1, j ; k}\right\}$ :

$$
\varphi_{j, k} \equiv\left|\begin{array}{ccccc}
T_{1, j, k-r} & T_{1, j-1, k+1-r} & \cdots & T_{1, j-r+1, k-1} & T_{1, j-r, k} \\
T_{1, j+1, k+1-r} & T_{1, j, k+2-r} & \cdots & T_{1, j-r+2, k} & T_{1, j-r+1, k+1} \\
\vdots & \vdots & \ddots & \vdots & \vdots \\
T_{1, j+r-1, k-1} & T_{1, j+r-2, k} & \cdots & T_{1, j, k+r-2} & T_{1, j-1, k+r-1} \\
T_{1, j+r, k} & T_{1, j+r-1, k+1} & \cdots & T_{1, j+1, k+r-1} & T_{1, j, k+r}
\end{array}\right|=1
$$

This is the "equation of motion" for the system. Since $\varphi_{j, k}$ is a discrete Wronskian determinant, it remains constant for solutions of a difference equation. The difference equation can be found by taking the difference of two Wronskians and arguing that a non-trivial linear combination of its columns must vanish.

Theorem 3.2. We have the following linear recursion relations

$$
\sum_{b=0}^{r+1} T_{1, j-b, k+b}(-1)^{b} c_{r+1-b}(j-k)=0 \quad j, k \in \mathbb{Z}
$$

where the coefficients $c_{r+1-b}(j-k)$ depend only on the difference $j-k$, with $c_{0}(m)=$ $c_{r+1}(m)=1$ for all $m \in \mathbb{Z}$, and:

$$
\sum_{a=0}^{r+1} T_{1, j+a, k+a}(-1)^{a} d_{r+1-a}(j+k)=0 \quad j, k \in \mathbb{Z}
$$

where the coefficients $d_{r+1-a}(j+k)$ depend only on the sum $j+k$, with $d_{0}(m)=d_{r+1}(m)=$ 1 for all $m \in \mathbb{Z}$.

Such linear recursion relations can be obtained by noting that $T_{r+2, j, k}=0$ and expanding the corresponding Wronskian determinant along the first row or column. The key fact to be proven is that the minors depend only on the difference $j-k$ or the sum $j+k$. The proof is presented in the Appendix, Section A.3.

By analogy with the case of the $Q$-systems $[6,7]$, we may still call the variables $c_{b}(k)$ and $d_{b}(k)$ integrals of motion of the $T$-system, as they depend on one less variable than $T$. Moreover, they can be expressed entirely in terms of the fundamental initial data for the $T$-system, $\widetilde{\mathbf{x}}_{0}$.

Example 3.3. In the $A_{1}$ case, we have

$$
\begin{aligned}
& T_{1, j, k}-c_{1}(j-k) T_{1, j-1, k+1}+T_{1, j-2, k+2}=0 \\
& T_{1, j, k}-d_{1}(j+k) T_{1, j+1, k+1}+T_{1, j+2, k+2}=0
\end{aligned}
$$


with the integrals of motion

$$
\begin{aligned}
& c_{1}(j)=\frac{T_{1, j, 0}}{T_{1, j-1,1}}+\frac{1}{T_{1, j-1,1} T_{1, j-2,0}}+\frac{T_{1, j-3,1}}{T_{1, j-2,0}} \\
& d_{1}(j)=\frac{T_{1, j, 0}}{T_{1, j+1,1}}+\frac{1}{T_{1, j+1,1} T_{1, j+2,0}}+\frac{T_{1, j+3,1}}{T_{1, j+2,0}}
\end{aligned}
$$

An explicit expression for the conserved quantities of Theorem 3.2 is as Wronskian determinants with a "defect":

Lemma 3.4. The conserved quantities $c_{m}(j)(m=0,1, \ldots, r+1, j \in \mathbb{Z})$ of Equation (3.3) are

$$
c_{m}(j)=\operatorname{det}_{\substack{1 \leqslant a \leqslant r+1 \\ 1 \leqslant b \leqslant r+2, b \neq r+2-m}}\left(T_{1, j+n+a-b, n+a+b-2}\right)
$$

for any $n \in \mathbb{Z}$.

Again the proof uses the standard techniques, and is found in Section A.4 of the Appendix.

\section{Conserved quantities and hard particles}

\subsection{Recursion relations for conserved quantities}

The conserved quantities (3.5) satisfy linear recursion relations, which allow us to express them in terms of the initial data $\mathbf{x}_{0}$. We use recursion relations on the size $r$, so we first relax the boundary conditions $T_{r+1, j, k}=1$ for all $j, k \in \mathbb{Z}$.

Consider the " $A_{\infty / 2}$ " ${ }^{1} T$-system:

$$
t_{\alpha, j, k+1} t_{\alpha, j, k-1}=t_{\alpha, j+1, k} t_{\alpha, j-1, k}+t_{\alpha+1, j, k} t_{\alpha-1, j, k}, \quad t_{0, j, k}=1,\left(j, k \in \mathbb{Z}, \alpha \in \mathbb{Z}_{>0}\right) .
$$

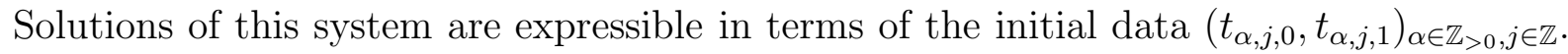
By definition, $T_{\alpha, j, k}=t_{\alpha, j, k}$ if we impose the boundary condition $t_{r+1, j, k}=1$ for all $j, k \in \mathbb{Z}$.

The proof of Theorem 3.1 does not involve the boundary condition $T_{r+1, j ; k}=1$, so the determinant expression for $t_{\alpha, j ; k}$ still holds:

$$
t_{\alpha, j, k}=\operatorname{det}_{1 \leqslant a, b \leqslant \alpha}\left(t_{1, j+a-b, k+a+b-\alpha-1}\right), \quad \alpha>1 .
$$

Define the Wronskians of size $N$ with a defect in position $N-m$ :

$$
c_{N, m, j, k}=\operatorname{det}_{\substack{1 \leqslant a \leqslant N \\ 1 \leqslant b \leqslant N+1, b \neq N+1-m}}\left(t_{1, j+a-b, k+a+b-N-1}\right),
$$

where $c_{N, m, j, k}=0$ if $m>N$ or $m<0$.

Note that $c_{N, 0, j, k}=T_{N, j, k}$, and $c_{N, N, j, k}=T_{N, j-1, k+1}$ by Theorem 3.1. If we impose the second boundary condition of the $T$-system on the $t$ 's, then $c_{r+1, m, j, k}=c_{m}(j-k+r)$.

\footnotetext{
${ }^{1}$ The notation $A_{\infty / 2}$ refers to the fact that the range of $\alpha$ is the positive integer half-line $[0, \infty)$, as opposed to $\mathbb{Z}$ for the case of $A_{\infty}$.
} 
Lemma 4.1. The Wronskians with a defect $c_{\alpha, m, j, k}$ satisfy the recursion relations:

$$
\begin{aligned}
t_{\alpha-1, j-1, k-1} c_{\alpha-1, m, j, k} & =t_{\alpha, j-1, k} c_{\alpha-2, m-1, j, k-1}+t_{\alpha-1, j, k} c_{\alpha-1, m, j-1, k-1} \\
t_{\alpha-1, j-1, k} c_{\alpha, m, j, k} & =t_{\alpha, j-1, k+1} c_{\alpha-1, m-1, j, k-1}+t_{\alpha, j, k} c_{\alpha-1, m, j-1, k}
\end{aligned}
$$

for $\alpha \geqslant 2$ and $m, j, k \geqslant 1$.

Proof. The first equation (4.4) follows from the Desnanot-Jacobi relation (A.3), with $N=\alpha, i_{1}=1, i_{2}=\alpha, j_{1}=\alpha-m, j_{2}=\alpha$, for the matrix $M$ with entries $M_{a, b}=$ $T_{1, j+a-b-1, k+a+b-\alpha-1}, a, b=1,2, \ldots, \alpha$.

The second equation (4.5) follows from the Plücker relation (A.2), with $N=\alpha$, and the $N \times(N+2)$ matrix $P$ with entries $P_{a, 1}=\delta_{a, \alpha}$, and $P_{a, b}=T_{1, j+a-b, k+a+b-\alpha-1}$ for $b=2,3, \ldots, \alpha+2$ and $a=1,2, \ldots, \alpha$, and by further picking $a_{1}=1, a_{2}=2, b_{1}=\alpha+2-m$, and $b_{2}=\alpha+2$.

Theorem 4.2. The Wronskians with a defect $c_{\alpha, m, j, k}$ defined in (4.3) are uniquely determined by the following recursion relation, for $\alpha \geqslant 2$ :

$$
\begin{aligned}
t_{\alpha-1, j-1, k-1} & t_{\alpha-1, j, k} c_{\alpha, m, j, k-1}=t_{\alpha-1, j-1, k-1} t_{\alpha, j-1, k} c_{\alpha-1, m-1, j+1, k-1} \\
+ & t_{\alpha, j, k-1} t_{\alpha-1, j, k} c_{\alpha-1, m, j-1, k-1}+t_{\alpha, j, k-1} t_{\alpha, j-1, k} c_{\alpha-2, m-1, j, k-1}
\end{aligned}
$$

and the boundary conditions $c_{0, m, j, k}=\delta_{m, 0}$, for all $j, k \in \mathbb{Z}$ and $c_{1, m, j, k}=\delta_{m, 0} t_{1, j, k}+$ $\delta_{m, 1} t_{1, j-1, k+1}$ for all $m, j, k \in \mathbb{Z}$.

Proof. Using Equation (4.4), the second line in (4.6) is equal to $t_{\alpha, j, k-1} t_{\alpha-1, j-1, k-1} c_{\alpha-1, m, j, k}$. Canceling the overall factor $t_{\alpha-1, j-1, k-1}$, we must prove that

$$
t_{\alpha-1, j, k} c_{\alpha, m, j, k-1}-\left(t_{\alpha, j-1, k} c_{\alpha-1, m-1, j+1, k-1}+t_{\alpha, j, k-1} c_{\alpha-1, m, j, k}\right)=0 .
$$

Multiplying the l.h.s. of (4.7) by $t_{\alpha, j+1, k}$ and using (4.1) we have

$$
\begin{aligned}
& t_{\alpha, j+1, k} t_{\alpha-1, j, k} c_{\alpha, m, j, k-1}-\left(t_{\alpha, j, k+1} t_{\alpha, j, k-1}-t_{\alpha+1, j, k} t_{\alpha-1, j, k}\right) c_{\alpha-1, m-1, j+1, k-1} \\
& -t_{\alpha, j+1, k} t_{\alpha, j, k-1} c_{\alpha-1, m, j, k} \\
= & t_{\alpha-1, j, k}\left(t_{\alpha, j+1, k} c_{\alpha, m, j, k-1}+t_{\alpha+1, j, k} c_{\alpha-1, m-1, j+1, k-1}\right) \\
& -t_{\alpha, j, k-1}\left(t_{\alpha, j, k+1} c_{\alpha-1, m-1, j+1, k-1}+t_{\alpha, j+1, k} c_{\alpha-1, m, j, k}\right) \\
= & t_{\alpha, j, k-1}\left(t_{\alpha-1, j, k} c_{\alpha, m, j+1, k}-t_{\alpha, j, k+1} c_{\alpha-1, m-1, j+1, k-1}-t_{\alpha, j+1, k} c_{\alpha-1, m, j, k}\right)=0
\end{aligned}
$$

where we have simplified the third line by use of (4.4), and finally used (4.5). Equation (4.6) follows.

Equation (4.6) is a three-term linear recursion relation in the variable $\alpha$ and therefore has a unique solution $c$ given the initial conditions at $\alpha=0,1$. Moreover, these initial conditions are identical to those for (4.3), hence this solution coincides with the definition (4.3) for all $\alpha, m, j, k$. 
Let us define:

$$
C_{\alpha+1, m}(j, k)=\frac{c_{\alpha+1, m, j+k-\alpha, k}}{t_{\alpha+1, j+k-\alpha, k}}, \quad \alpha \geqslant 0, \quad m, j, k \in \mathbb{Z} .
$$

These satisfy $C_{\alpha+1,0}(j, k)=1$ and $C_{\alpha+1, \alpha+1}(j, k)=t_{\alpha+1, j+k-\alpha-1, k+1} / t_{\alpha+1, j+k-\alpha, k}$. The conserved quantities of the $T$-system of type $A_{r}$ are obtained by imposing the boundary condition $t_{r+1, j, k}=1$, in which case: $c_{m}(j)=C_{r+1, m}(j, k)$ for any $j \in \mathbb{Z}$, and $m=$ $0,1,2, \ldots, r+1$, independently of $k \in \mathbb{Z}$.

Corollary 4.3. The quantities $C_{\alpha+1, m}(j, k)$ of eq.(4.8) are the solutions of the following linear recursion relation, for $\alpha \geqslant 1$ :

$$
\begin{aligned}
C_{\alpha+1, m}(j, k)= & C_{\alpha, m}(j-2, k)+y_{2 \alpha+1}(j-\alpha, k) C_{\alpha, m-1}(j, k) \\
& +y_{2 \alpha}(j-\alpha-1, k) C_{\alpha-1, m-1}(j-2, k)
\end{aligned}
$$

with coefficients:

$$
\begin{aligned}
y_{2 \alpha+1}(j, k) & =\frac{t_{\alpha+1, j+k-1, k+1} t_{\alpha, j+1+k, k}}{t_{\alpha+1, j+k, k} t_{\alpha, j+k, k+1}} \quad(\alpha \geqslant 1) \\
y_{2 \alpha}(j, k) & =\frac{t_{\alpha+1, j+k, k+1} t_{\alpha-1, j+k+1, k}}{t_{\alpha, j+k, k} t_{\alpha, j+k+1, k+1}} \quad(\alpha \geqslant 1) \\
y_{1}(j, k) & =\frac{t_{1, j+k, k+1}}{t_{1, j+k+1, k}},
\end{aligned}
$$

subject to the initial conditions $C_{0, m}(j, k)=\delta_{m, 0}$ and $C_{1, m}(j, k)=\delta_{m, 0}+\delta_{m, 1} y_{1}(j-1, k)$.

Example 4.4. We have the following first few values of $C_{\alpha+1, m}(j, k)$ :

$$
\begin{aligned}
\alpha=0: & C_{1,0}(j, k)=1 \\
& C_{1,1}(j, k)=y_{1}(j-1, k) \\
\alpha=1: & C_{2,0}(j, k)=1 \\
& C_{2,1}(j, k)=y_{1}(j-3, k)+y_{2}(j-2, k)+y_{3}(j-1, k) \\
& C_{2,2}(j, k)=y_{1}(j-1, k) y_{3}(j-1, k) \\
\alpha=2: & C_{3,0}(j, k)=1 \\
C_{3,1}(j, k)= & y_{1}(j-5, k)+y_{2}(j-4, k)+y_{3}(j-3, k)+y_{4}(j-3, k)+y_{5}(j-2, k) \\
C_{3,2}(j, k)= & y_{1}(j-3, k) y_{3}(j-3, k)+y_{4}(j-3, k) y_{1}(j-3, k) \\
& +y_{5}(j-2, k)\left(y_{1}(j-3, k)+y_{2}(j-2, k)+y_{3}(j-1, k)\right) \\
& C_{3,3}(j, k)=y_{1}(j-1, k) y_{3}(j-1, k) y_{5}(j-2, k)
\end{aligned}
$$

Remark 4.5. The Corollary 4.3 allows to interpret the conserved quantities of the $T$ system of type $A_{r}$ as follows. From the recursion relation (4.9), we deduce that $C_{r+1, m}(j, k)$ is a homogeneous polynomial of the weights $y_{1}, y_{2}, \ldots, y_{2 r+1}$, themselves ratios of products of some $t_{a, b, c}$ 's with $c$ only taking the values $k$ and $k+1$. If we impose $t_{r+1, j, k}=1$, we 


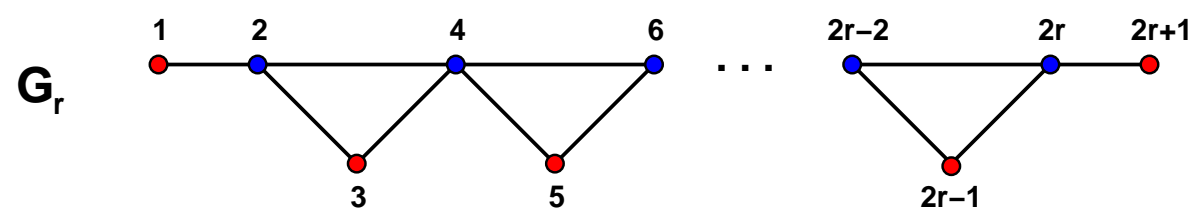

Figure 4.1: The graph $G_{r}$, with $2 r+1$ vertices labeled $i=1,2, \ldots, 2 r+1$.

see that, as explained above, $C_{r+1, m}(j, k)=c_{m}(j)$ is independent of $k$. We may therefore write $C_{r+1, m}(j, k)=C_{r+1, m}(j, 0)$, the latter involving only $T_{a, b, c}$ 's with $c=0,1$. These give $r$ conservation laws for $m=1,2, \ldots, r$. For $r=1$, we have for instance

$$
\begin{aligned}
C_{2,1}(j, k) & =\frac{T_{1, j+k, k}}{T_{1, j+k-1, k+1}}+\frac{1}{T_{1, j+k-1, k+1} T_{1, j+k-2, k}}+\frac{T_{1, j+k-3, k+1}}{T_{1, j+k-2, k}} \\
& =C_{2,1}(j, 0)=\frac{T_{1, j, 0}}{T_{1, j-1,1}}+\frac{1}{T_{1, j-1,1} T_{1, j-2,0}}+\frac{T_{1, j-3,1}}{T_{1, j-2,0}}
\end{aligned}
$$

\subsection{Hard particle interpretation}

In this paper, we introduce a slightly generalized model of hard particles on a graph.

\subsubsection{Definition of the model}

Let $G_{r}$ be the graph of Figure 4.1, with vertices labeled as shown. When $r=1, G_{1}$ is just the chain with 3 vertices, and when $r=0 G_{0}$ is a single vertex.

To each vertex labeled $i$ in $G_{r}$, we associate a height function $h$, where

$$
h(i)=\left\lfloor\frac{i+1}{2}\right\rfloor,(i>1), h(1)=0 .
$$

A configuration of hard particles on $G_{r}$ is a subset $S$ of $I_{2 r+1}$ such that $i, j \in I$ implies that vertices $i$ and $j$ are not connected by an edge. We can think of the elements of $I$ as the vertices occupied by particles. The set of all hard particle configurations of cardinality $m$ on $G_{r}$ is called $\mathcal{C}_{m}$. There is a natural ordering on the set $I_{2 r+1}$, and in the generalized hard particle model we define in this paper, the set $S$ is considered to be an ordered set.

In general, a hard particle model on $G_{r}$ associates weights to the occupied vertices which depend on the vertex label, and possibly also on the total number of occupied particles. The corresponding partition function is the sum over all possible hard-particle configurations of the products of the occupied vertex weights.

For the purpose of this work, we define the partition function for $m$ hard particles as

$$
Z_{m}^{G_{r}}(j, k)=\sum_{S \in \mathcal{C}_{m}} \prod_{\ell=1}^{m} y_{i_{\ell}}\left(j-2(r+\ell-m)-1+h\left(i_{\ell}\right), k\right)
$$

with the weights $y_{i}$ as in (4.10) and $S=\left\{i_{1}, \ldots, i_{m}\right\}$. 


\subsubsection{Conserved quantities as hard particle partition functions}

We have the following.

Theorem 4.6. The partition function $Z_{m}^{G_{\alpha}}(j, k)(4.11)$ for $m$-hard particles on $G_{\alpha}$ coincides with the quantity $C_{\alpha+1, m}(j, k)$ of $(4.8)$.

Proof. Hard particle partition functions on $G_{r}$ satisfy a recursion relation in $r$. Fix $m$ and consider the configuration of particles on vertices $(2 r+1,2 r)$. There are 3 possible pairs of occupation numbers for these two neighboring vertices, $(0,0),(1,0)$ and $(0,1)$, respectively contributing to the partition function:

- $(0,0)$ contributes $Z_{m}^{G_{r-1}}(j-2, k)$.

- $(1,0)$ contributes $y_{2 r+1}(j-r, k) Z_{m-1}^{G_{r-1}}(j, k)$.

- $(0,1)$ contributes $y_{2 r}(j-r-1, k) Z_{m-1}^{G_{r-2}}(j-2, k)$.

This implies that $Z_{m}^{G_{r}}$ satisfies the recursion relation

$Z_{m}^{G_{r+1}}(j, k)=Z_{m}^{G_{r}}(j-2, k)+y_{2 r+1}(j-r, k) Z_{m-1}^{G_{r}}(j, k)+y_{2 r}(j-r-1, k) Z_{m-1}^{G_{r-1}}(j-2, k)$.

But this is the same relation satisfied by $C_{r, m}(j, k)$, Equation (4.9), with the same initial conditions, $Z_{0}^{G_{r}}(j, k)=1$ (for any $r$ ) and and $Z_{1}^{G_{0}}(j, k)=y_{1}(j-1, k)=C_{1,1}(j, k)$. The theorem follows.

Setting $t_{r+1, j, k}=1$, we have:

Corollary 4.7. The conserved quantities $c_{m}(j)$ of the $T$-system of type $A_{r}$ are the partition functions for $m$-hard particles on $G_{r}$, with the weights:

$$
\begin{aligned}
y_{2 \alpha+1}(j, k) & =\frac{T_{\alpha+1, j+k-1, k+1} T_{\alpha, j+1+k, k}}{T_{\alpha+1, j+k, k} T_{\alpha, j+k, k+1}} \quad(1 \leqslant \alpha \leqslant r) \\
y_{2 \alpha}(j, k) & =\frac{T_{\alpha+1, j+k, k+1} T_{\alpha-1, j+k+1, k}}{T_{\alpha, j+k, k} T_{\alpha, j+k+1, k+1}} \quad(1 \leqslant \alpha \leqslant r) \\
y_{1}(j, k) & =\frac{T_{1, j+k, k+1}}{T_{1, j+k+1, k}}
\end{aligned}
$$

where $T_{0, j, k}=T_{r+1, j, k}=1$ for all $j, k \in \mathbb{Z}$.

As the resulting hard-particle partition functions are independent of $k$, we may set $k=0$ in the expression for the weights. 


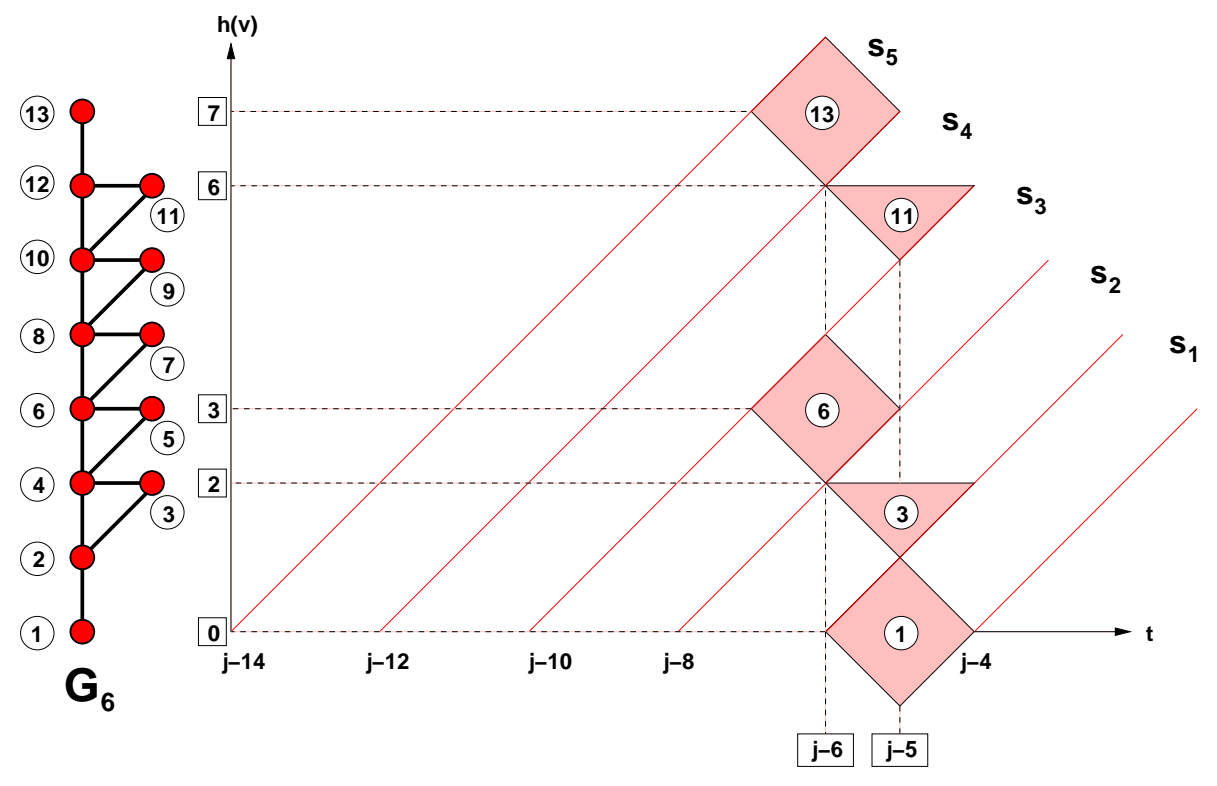

Figure 4.2: A graphical interpretation of a hard-particle configuration on the graph $G_{6}$, with $m=5$ particles at positions $\{1,3,6,11,13\}$. The label of the occupied vertex is indicated in a circle, and the time and height coordinates in rectangles. A distinct diagonal stripe corresponds to each particle. The leftmost stripe has $x$-intercept $j-2(r+1)=j-14$, and the rightmost is at $j-2(r+1-m)=j-4$. The weight of this configuration is $y_{1}(j-5, k) y_{3}(j-5, k) y_{6}(j-$ $6, k) y_{11}(j-5, k) y_{13}(j-6, k)$.

\subsection{A pictorial representation for the hard particle partition function}

The hard particle configurations which give rise to the partition function of the form (4.11) can be represented graphically as in Figure 4.2.

- A particle at a spine vertex $v(v \in\{1,2,4,6, \ldots, 2 r-2,2 r, 2 r+1\})$ is represented by a diamond on the two-dimensional lattice, its center at the height of the vertex, at the point $(t, h(v))$ for some $t \in \mathbb{Z}$, and its vertices at the four neighboring lattice sites.

- A particle a vertex $v \in\{3,5,7, \ldots, 2 r-1\}$ is represented by the lower half of such a diamond.

We call $t$ the time coordinate, and $h(v)$ the height. Each polygon is at $(t, h(v))$ contained in a diagonal stripe $s$, bordered by the lines $y=x-(t+1-h(v))$ and $y=x-(t-1-h(v))$. We denote $s$ by its $x$-intercepts, $s=\{t-1-h(v), t+1-h(v)\}$.

Given a configuration $S \in \mathrm{C}_{m}$, with $S=\left\{i_{1}<i_{2}<\cdots<i_{m}\right\}$, the polygon representing the particle $i_{1}$ is drawn in the stripe $s_{1}=\{t-2, t\}$; that of $i_{2}$ in the stripe immediately above and to the left, $s_{2}=\{t-4, t-2\}$, and the $k$-th polygon representing $i_{k}$ lies in stripe $s_{k}=\{t-2 k, t-2 k+2\}$. The height of each polygon is determined by $h\left(i_{j}\right)$ and its time coordinate by its stripe: $t_{k}=h\left(i_{k}\right)-1+t-2(k-1)$. 


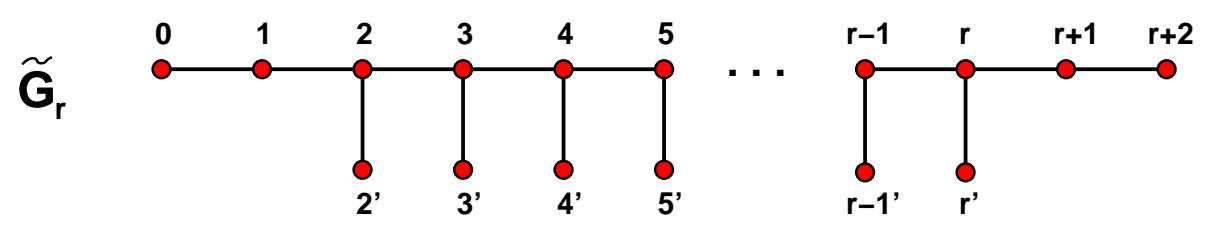

Figure 5.1: The graph $\widetilde{G}_{r}$, with $2 r+2$ vertices.

If we choose $t=j-2(r+1-m)$, then Equation (4.11) can be written as

$$
Z_{m}^{G_{r}}(j, k)=\sum_{S \in \mathfrak{C}_{m}} \prod_{\ell=1}^{m} y_{i_{\ell}}\left(t_{\ell}, k\right)
$$

\section{Path formulation and positivity}

In this section we provide an expression for $T_{\alpha, j, k}$ as a function of the initial data $\mathbf{x}_{0}=$

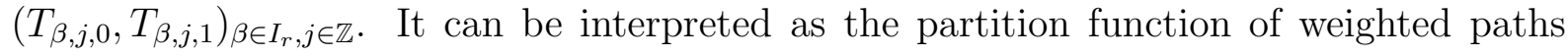
on a certain graph, with time-dependent weights. That is, we generalize the notion of a weighted path, so that the weight of a step in the path depends on the time at which it is taken.

As a corollary of the formulation in this section, we have the positivity Theorem 5.7 for the variables $T_{\alpha, j, k}$ as a function of the initial data.

\subsection{Definitions}

Let $\widetilde{G}_{r}$ be the graph in Figure 5.1. It has $2 r+2$ vertices, which are ordered as $0,1,2,2^{\prime}$, $3,3^{\prime}, \ldots r, r^{\prime}, r+1, r+2$. Its incidence matrix $A$ is

$$
A_{m, m^{\prime}}=A_{m^{\prime}, m}=1,(2 \leqslant m \leqslant r) ; \quad A_{m, m+1}=A_{m+1, m}=1,(0 \leqslant m \leqslant r+1) .
$$

The vertex labelled 0 is called the origin of the graph. We call the vertices $i$ the spine vertices of $\widetilde{G}_{r}$, and the edges which connect $i \rightarrow 1 \pm 1$ spine edges.

We consider the set $\mathcal{P}_{t_{1}, t_{2}}^{a, b}$ of paths $p$ on the graph $\tilde{G}_{r}$, starting at time $t_{1}$ and vertex $a$, and ending at time $t_{2} \geqslant t_{1}$ at vertex $b$. We take $t_{i} \in \mathbb{Z}$, and each step takes one time unit. The path $p$ may be represented by the succession of visited vertices, $p=(p(t))_{t=t_{1}, t_{1}+1, \ldots, t_{2}}$, with $p\left(t_{1}\right)=a$ and $p\left(t_{2}\right)=b$ and $A_{p(s), p(s+1)}=1$ for any $s$.

Let $w_{i, j}(t)$ be the weight of a step vertex $i$ to vertex $j$ at time $t$. We define the weight of a path $p \in \mathcal{P}_{t_{1}, t_{2}}^{a, b}$ to be

$$
w(p)=\prod_{s=t_{1}}^{t_{2}-1} w_{p(s), p(s+1)}(s), \quad p\left(t_{1}\right)=a, p\left(t_{2}\right)=b .
$$

The partition function for weighted paths in $\mathcal{P}_{t_{1}, t_{2}}^{a, b}$ is

$$
z_{t_{1}, t_{2}}^{a, b}=\sum_{p \in \mathcal{P}_{t_{1}, t_{2}}^{a, b}} w(p)
$$




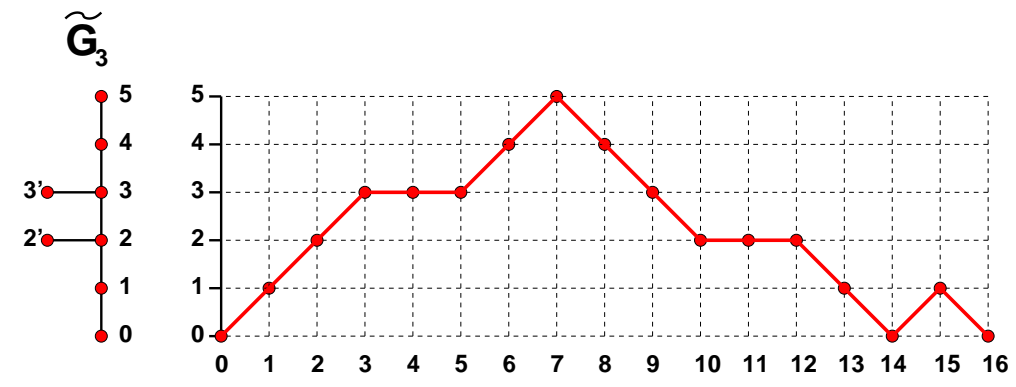

Figure 5.2: The planar representation of a typical path in $\mathcal{P}_{0,16}^{0,0}$ on the graph $\tilde{G}_{3}$.

For later use, we define $z_{t_{1}, t_{2}}^{a, b}=0$ if $t_{1}>t_{2}$.

Paths can be represented on the lattice $\mathbb{Z}^{2}$ as in Figure 5.2. We associate a vertical coordinate $h(i)=h\left(i^{\prime}\right)=i$ to each vertex of $\widetilde{G}_{r}$. The horizontal axis is the time. A step $a \rightarrow b$ at time $t$ on $\widetilde{G}_{r}$ is a step $(t, h(a)) \rightarrow(t+1, h(b))$.

We claim (see Theorem 5.5) that there exists a choice of weights $w_{a, b}(s)$, as functions

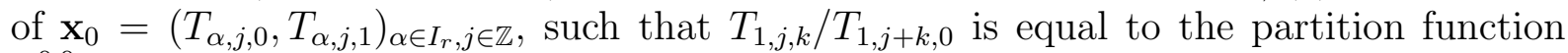
$z_{j-k, j+k}^{0,0}$.

Dividing a path, which takes place from time $t$ to time $t^{\prime}$, into a first part from $t$ to $t^{\prime}$, and a second part, from $t^{\prime}$ to $t^{\prime \prime}$, we have

$$
z_{t, t^{\prime}}^{a, b}=\sum_{x \in \widetilde{G}_{r}} z_{t, t^{\prime}}^{a, x} z_{t^{\prime}, t^{\prime \prime}}^{x, b}, \quad t^{\prime} \in\left[t, t^{\prime \prime}\right]
$$

In particular, the matrix of one-step partition functions $z_{t, t+1}^{a, b}$ is called the transfer matrix $\mathcal{T}(t)$, with entries

$$
(\mathcal{T}(t))_{a, b}=z_{t, t+1}^{a, b}=w_{a, b}(t) A_{a, b}
$$

The transfer matrix is a decorated adjacency matrix. The recursion relation (5.3) implies

$$
z_{t_{1}, t_{2}}^{a, b}=\left(\mathcal{T}\left(t_{1}\right) \mathcal{T}\left(t_{1}+1\right) \cdots \mathcal{T}\left(t_{2}-1\right)\right)_{a, b}
$$

We use the following definition for weights $w_{a, b}(s)$ of paths on $\widetilde{G}_{r}$ :

$$
\begin{aligned}
& w_{m, m^{\prime}}(s)=1, \quad w_{m^{\prime}, m}(s)=y_{2 m+1}(s, 0),(m \in\{1, \ldots, r\}), \\
& w_{m, m+1}(s)=1, \quad w_{m+1, m}(s)=y_{2 m}(s, 0), \quad(m \in\{1, \ldots, r-1\}), \\
& w_{0,1}(s)=1, \quad w_{1,0}(s)=y_{1}(s, 0),
\end{aligned}
$$

in terms of the weights $y_{i}(s, k)$ of Equation (4.13).

\subsection{An involution on pairs of weights}

We define an involution $\varphi$ on the set $\mathcal{C}_{m} \times \mathcal{P}_{j-2(r+1-m), j+2 k}^{0,0}$, consisting of hard-particle configurations on $G_{r}$ and paths on $\widetilde{G}_{r}$. 

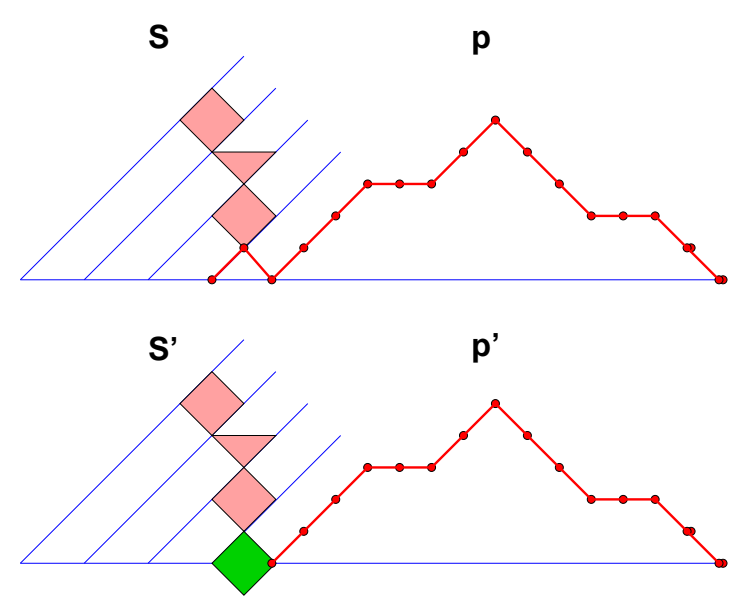

(a)
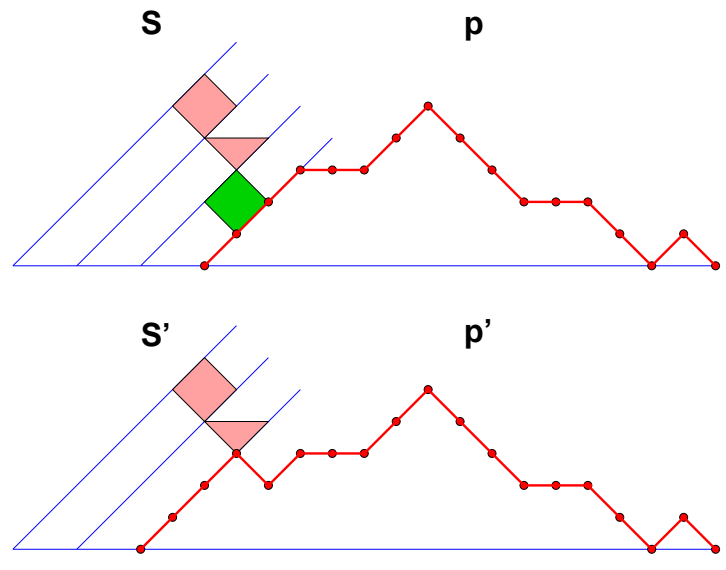

(b)

Figure 5.3: The involution $\varphi$ between pairs of $m$ - hard particle configurations and paths in $\mathcal{P}_{j-2(r+1-m), j+2 k}^{0,0}$. Case (a): the first stripe traversed by $p$ is absorbed into $S^{\prime}$, which has $m+1$ particles, and $p^{\prime} \in \mathcal{P}_{j-2(r+1-m)+2, j+2 k}^{0,0}$. Case (b): The bottom stripe of $S$ is absorbed into $p^{\prime}$, now in $\mathcal{P}_{j-2(r+1-m)-2, j+2 k}^{0,0}$, while $S^{\prime}$ has only $m-1$ particles.

Let $(S, p) \in \mathcal{C}_{m} \times \mathcal{P}_{j-2(r+1-m), j+2 k}^{0,0}$, with $m \in\{0, \ldots, r+1\}, k \in \mathbb{Z}_{+}$. We refer to the graphical representations of Figures 4.2 and 5.2, and we draw $S$ and $p$ on the same lattice (see Figure 5.3), where $S$ is represented between the diagonal lines $y=x-(j-2(r+1))$ and $y=x-(j-2(r+1-m))$, and $p$ starts at $(j-2(r+1-m), 0)$, the $x$-intercept of the bottom stripe of $S$.

The path $p$ has an initial section $p_{0}$ within the diagonal stripe $\{j-2(r+1-m), j-$ $2(r-m)\}$, consisting of $u$ consecutive up steps and (i) a down step $(s, u) \rightarrow(s+1, u-1)$ or (ii) two horizontal steps $(s, u) \rightarrow(s+1, u) \rightarrow(s+2, u)$, where $s=j-2(r+1-m)+u$. $p \backslash p_{0}$ is then to the right of this initial stripe.

Let $\sigma$ be a map from path steps of type (i) or (ii) on $\widetilde{G}_{r}$ to the vertex set of $G_{r}$. It is defined as follows:

$$
\begin{aligned}
\sigma((s, u) \rightarrow(s+1, u-1)) & = \begin{cases}2 u-2, & 2 \leqslant u \leqslant r+1 \\
1, & u=1 \\
2 r+1, & u=r+2 .\end{cases} \\
\sigma((s, u) \rightarrow(s+2, u)) & =2 u-1 .
\end{aligned}
$$

Remark 5.1. Graphically, steps of type (i) and (ii) in p can mapped precisely to the polygons representing particles on $G_{r}$. A step of type (i) is the NE edge of a diamond (hence a particle on a spine vertex) and a step of type (ii) the upper edge of a halfdiamond. The map $\sigma$ represents this correspondence.

Denote by $i$ the image of a step under the map $\sigma$. We must now distinguish between two cases. 
- Case (a): If $i<i_{1}$ and $S^{\prime}:=\left\{i, i_{1}, i_{2}, \ldots, i_{m}\right\} \subset \mathcal{C}_{m+1}$, define $p^{\prime} \in \mathcal{P}_{j-2(r+1-m)+2, j+2 k}^{0,0}$ to be the path with $p^{\prime}(j-2(r+1-m)+2+x)=x$ for $x=0,1, \ldots, u$ (case $\left.(\mathrm{i})\right)$ or $x=0,1, \ldots, u-1$ (case (ii)), and $p^{\prime}(x)=p(x+2)$ otherwise.

- Case (b): If $i \geqslant i_{1}$ or $\left\{i, i_{1}, \ldots, i_{m}\right\} \notin \mathcal{C}_{m+1}$, define $S^{\prime}=\left\{i_{2}, i_{3}, \ldots, i_{m}\right\} \in \mathcal{C}_{m-1}$, the hard particle configuration with the right stripe removed. It is now drawn between the diagonal lines $y=x-(j-2(r+1))$ and $y=x-(j-2(r+1-(m-1)))$. As for the path $p^{\prime}$,

- If $i_{1} \in\{3,5, \ldots, 2 r-1\}$, define $p^{\prime}(j-2(r+2-m)+x)=x$ for $x=0,1, \ldots, h\left(i_{1}\right)$, $p^{\prime}\left(j-2(r+2-m)+h\left(i_{1}\right)+y\right)=h\left(i_{1}\right)$ for $y=1,2$.

- Otherwise, $p^{\prime}(j-2(r+2-m)+x)=x$ for $x=0,1, \ldots, h\left(i_{1}\right)+1$, and $p^{\prime}(j-$ $\left.2(r+2-m)+h\left(i_{1}\right)+2\right)=h\left(i_{1}\right)$.

In both cases, $p^{\prime}(x)=p(x-2)$ for the remaining times.

Remark 5.2. Graphically the map can be visualized as follows. If the particle represented by $p_{0}$ can be added to $S$ while keeping the hard-particle condition, then we do this, while changing $p_{0}$ so that it consists only of up steps, starting two steps to the right of the original starting point of $p$. Otherwise, perform the opposite operation, changing the first particle to a path segment.

In view of the graphical description, the map $\varphi$ is clearly an involution. Moreover it is weight-preserving: In Equation (5.6), only the steps of type (i) or (ii) have a non-trivial weight. Moreover, $w(\sigma($ step $))=w($ step) according to Equation (4.13) (setting $k=0$ ). Therefore, $w(S, p)=w(S) w(p)=w\left(S^{\prime}\right) w\left(p^{\prime}\right)=w\left(S^{\prime}, p^{\prime}\right)$. We have

\section{Lemma 5.3.}

$$
\sum_{m=0}^{r+1}(-1)^{r+1-m} Z_{m}^{G_{r}}(j, 0) Z_{j-2(r+1-m), j+2 k}^{0,0}=0, \quad(j \in \mathbb{Z}, k \geqslant 0) .
$$

Proof. This is the partition function for pairs $(S, p) \in \cup_{m=0}^{r+1} \mathcal{C}_{m} \times \mathcal{P}_{j-2(r+1-m), j+2 k}^{0,0}$, with an extra factor $(-1)^{r+1-m}$ which ensures that the contributions of $(S, p)$ and $\varphi(S, p)$ cancel each other.

We can also consider the sum in Equation (5.7) in the case where $k<0$. The sum is non-trivial in those cases only if $k \geqslant-r-1$, since $z_{t, t^{\prime}}^{a, b}=0$ if $t>t^{\prime}$. We extend the definition of $\varphi: \varphi(S, p)=(S, p)$ if $S=\emptyset$ or if the path $p$ has length zero and $i_{1}>1$.

\section{Lemma 5.4.}

$$
\sum_{m=0}^{r+1-i}(-1)^{r+1-m} Z_{m}^{G_{r}}(j, 0) Z_{j-2(r+1-m), j-2 i}^{0,0}=(-1)^{i} Z_{r+1-i}^{G_{r}^{\prime}}(j, 0)
$$

where $Z_{m}^{G_{r}^{\prime}}(j, k)$ is the partition function of $m$ hard particles on $G_{r}^{\prime}$, the graph $G_{r}$ with vertex 1 removed (or the contribution to $Z_{m}^{G_{r}}$ in which vertex 1 is unoccupied). 
Proof. We apply the involution argument in the previous Lemma for $k$ in the range $-r-1 \leqslant k<0$. Pairs $(S, p)$ which are not invariant under $\varphi$ cancel each other. We are left with the contribution of the invariant pairs. The latter always have $p=\emptyset$ and the vertex 1 unoccupied.

Equations (5.8) are an expression for the initial conditions of the partition functions $z_{j-2(r+1-m), j-2 i}^{0,0}$ with $1 \leqslant i \leqslant r-1$ in terms of hard-particle partition functions.

\subsection{The $T$-system solution $T_{1, j, k}$ as a partition function of paths}

Our main result in this section is the following.

\section{Theorem 5.5.}

$$
T_{1, j, k}=T_{1, j+k, 0} z_{j-k, j+k}^{0,0} .
$$

Proof. We will show that $S_{j, k}=T_{1, j+k, 0} z_{j-k, j+k}^{0,0}$ satisfies the linear recursion relation (3.3) and coincides with $T_{1, j, k}$ when $k \in\{0, \ldots, r\}$ for any $j \in \mathbb{Z}$. Given that (3.3) has $r+1$ terms, this implies $S_{j, k}=T_{1, j, k}$ for all other $k$.

The sum

$$
\sum_{m=0}^{r+1}(-1)^{m} c_{r+1-m}(j-k) Z_{j-k-2 m, j+k}^{0,0}
$$

is equal to the sum in Equation (5.7), since $Z_{m}^{G_{r}}(j, 0)=c_{m}(j)$. Therefore, it vanishes for all $j \in \mathbb{Z}$ and $k \in \mathbb{Z}_{+}$. This implies that $S_{j, k}$ satisfies the same recursion relation (3.3) as $T_{1, j, k}$.

As for the initial conditions, we see from Equation (5.8) that $S_{j, k}$ satisfies

$$
\sum_{m=0}^{i}(-1)^{m} Z_{i-m}^{G_{r}}(j, 0) S_{j-m-2(r+1-i), m}=Z_{i}^{G_{r}^{\prime}}(j, 0) T_{1, j-2(r+1-i), 0} .
$$

We will show that the variables $T_{1, j, k}$ satisfy the same relations. Let

$$
W_{i}^{G_{\alpha}}(j)=\sum_{m=0}^{i}(-1)^{m} Z_{i-m}^{G_{\alpha}}(j, 0) \frac{T_{1, j-2(\alpha+1-i)-m, m}}{T_{1, j-2(\alpha+1-i), 0}}, \quad(0 \leqslant i \leqslant \alpha+1)
$$

Using the recursion relations (4.12), we find

$$
W_{i}^{G_{\alpha+1}}(j)=W_{i}^{G_{\alpha}}(j-2)+y_{2 \alpha+1}(j-\alpha) W_{i-1}^{G_{\alpha}}(j)+y_{2 \alpha}(j-\alpha-1) W_{i-1}^{G_{\alpha-1}}(j-2) .
$$

This is identical to the recursion relations (4.12) for $Z_{i}^{G_{\alpha}}(j, 0)$. Comparing the initial terms, we find that $W_{0}^{G_{0}}(j)=1$ and $W_{1}^{G_{0}}(j)=Z_{1}^{G_{0}}(j, 0)-\frac{T_{1, j-1,1}}{T_{1, j, 0}} Z_{0}^{G_{0}}(j, 0)=0$, so that $W_{i}^{G_{0}}(j)=\left.Z_{i}^{G_{0}}(j, 0)\right|_{y_{1}(j-1)=0}$. Moreover,

$$
\begin{aligned}
& W_{0}^{G_{1}}(j)=1 \\
& W_{1}^{G_{1}}(j)=Z_{1}^{G_{1}}(j, 0)-\frac{T_{1, j-3,1}}{T_{1, j-2,0}} Z_{0}^{G_{0}}(j, 0)=\left.Z_{1}^{G_{1}}(j, 0)\right|_{y_{1}(j-3)=0} \\
& W_{2}^{G_{1}}(j)=Z_{2}^{G_{1}}(j, 0)-\frac{T_{1, j-1,1}}{T_{1, j, 0}} Z_{1}^{G_{1}}(j, 0)+\frac{T_{1, j-2,2}}{T_{1, j, 0}} Z_{0}^{G_{1}}(j, 0)=0
\end{aligned}
$$


where we have used the identity between $Z$ and $C$, Example 4.4, the definitions (4.13), and $T$-system relations. In short, we have $W_{i}^{G_{\alpha}}(j)=\left.Z_{i}^{G_{\alpha}}(j, 0)\right|_{y_{1}=0}$, valid for all initial data $\alpha=0,1$. This implies

$$
W_{i}^{G_{\alpha}}(j)=\left.Z_{i}^{G_{\alpha}}(j, 0)\right|_{y_{1}=0} \text { for all } \alpha
$$

Thus, $W_{i}^{G_{\alpha}}(j)$ is the partition function for $i$ hard particles on $G_{\alpha}$ with weight 0 on vertex 1 , or alternatively, vertex 1 unoccupied. Therefore, $W_{i}^{G_{r}}(j)=Z_{i}^{G_{r}^{\prime}}(j, 0)$.

Therefore, $T_{1, j, k}$ and $S_{j, k}$ satisfy the same recursion relation and have the same boundary conditions. The Theorem follows.

The reasoning of Theorem 5.5 can be carried through by considering paths with weights $y_{\alpha}(j)$ replaced by the weights $y_{\alpha}(j, k)$ of eq.(4.10). We therefore have the following.

Corollary 5.6. If we define the weights $w_{a, b}(s, p)$ as follows:

$$
\begin{aligned}
& w_{m, m^{\prime}}(s, p)=1, \quad w_{m^{\prime}, m}(s, p)=y_{2 m+1}(s, p), \quad(m \in\{1, \ldots, r\}), \\
& w_{m, m+1}(s, p)=1, \quad w_{m+1, m}(s, p)=y_{2 m}(s, p), \quad(m \in\{1, \ldots, r-1\}), \\
& w_{0,1}(s, p)=1, \quad w_{1,0}(s, p)=y_{1}(s, p),
\end{aligned}
$$

with the $y_{i}(s, p)$ as in (4.13), then the following identity holds:

$$
T_{1, j, k}=T_{1, j+p, k-p} Z_{j-p, j+p}^{0,0}\left(\left\{y_{\alpha}(s, k-p), j-p \leqslant s \leqslant j+p, 1 \leqslant \alpha \leqslant 2 r+1\right\}\right) .
$$

Proof. We may view this as a particular case of the translational invariance $T_{\alpha, j, k} \rightarrow$ $T_{\alpha, j, k+1}$ of the $T$-system, namely that $T_{\alpha, j, k}$ is expressed as the same function of the initial

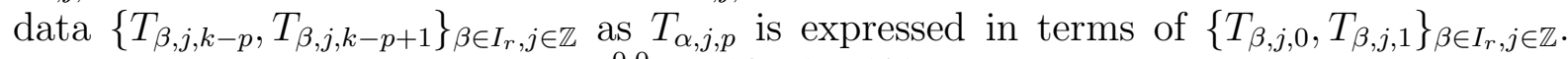
We deduce that $T_{1, j, p}=T_{1, j+p, 0} Z_{j-p, j+p}^{0,0}\left(\left\{y_{\alpha}(s, 0)\right\}\right)$ has the same expression as $T_{1, j, k}=$ $T_{1, j, p+k-p}$ in terms of $y_{\alpha}(s, k-p)$, and the corollary follows, as the prefactor itself comes from the substitution $T_{1, j+p, 0} \rightarrow T_{1, j+p, k-p}$.

\subsection{General $T$-system solution $T_{\alpha, j, k}$ : families of non- intersect- ing paths}

We may interpret $T_{\alpha, j, k}$ directly in terms of paths by use of the determinant expression of Theorem 3.1 for $T_{\alpha, j, k}$ in terms of the $T_{1, \ell, m}$. Indeed, given weighted paths on an acyclic graph $\Gamma$, say with partition function $z_{s, e}$ for paths starting at vertex $s$ and ending at vertex $e$, the Lindström-Gessel-Viennot formula gives an expression for the partition function of $\alpha$ non-intersecting paths on $\Gamma$ (i.e. such that no to paths share a vertex) as $z_{s_{1}, \ldots, s_{\alpha} ; e_{1}, \ldots, e_{\alpha}}=\operatorname{det}_{1 \leqslant i, j \leqslant \alpha} z_{s_{i}, e_{j}}$. We obtain:

$$
\begin{aligned}
T_{\alpha, j, k} & =\operatorname{det}_{1 \leqslant a, b \leqslant \alpha}\left(T_{1, j+k+2 b-\alpha-1,0} z_{j-k+\alpha+1-2 a, j+k+2 b-\alpha-1}^{0,0}\right) \\
& =\left(\prod_{b=1}^{\alpha} T_{1, j+k+2 b-\alpha-1,0}\right) z_{s_{1}, \ldots, s_{\alpha} ; e_{1}, \ldots, e_{\alpha}}^{0,0}
\end{aligned}
$$


where $Z_{s_{1}, \ldots, s_{\alpha} ; e_{1}, \ldots, e_{\alpha}}^{0,0}$ stands for the partition function of families of $\alpha$ non-intersecting paths in the plane representation of Section 5.1, starting at the points $s_{a}=(j-k+2 a-$ $\alpha-1,0), a=1,2, \ldots, \alpha$ and ending at the points $e_{b}=(j+k+\alpha+1-2 b, 0), b=1,2, \ldots, \alpha$. Alternatively, one may think of the partition function $z_{s_{1}, \ldots, s_{\alpha} ; e_{1}, \ldots, e_{\alpha}}$ as that of $\alpha$ "vicious" walkers (i.e. never meeting at a vertex) on $\tilde{G}_{r}$, going from the root to the root, respectively starting at times $j-k+2 a-\alpha-1$ and ending at times $j+k+\alpha+1-2 a, a=1,2, \ldots, \alpha$, each step corresponding to a unit of time.

Interpreted in this way, the $T_{\alpha, j, k}$ are manifestly positive Laurent polynomials of the initial data, via the weights $y_{\beta}(t)$ and the prefactor in (5.14). We therefore have the:

Theorem 5.7. The solution $T_{\alpha, j, k}$ of the T-system is expressed as a positive Laurent polynomial of the initial data $\mathbf{x}_{0}=\left\{T_{\beta, j, 0}, T_{\beta, j, 1}\right\}_{\beta \in I_{r}, j \in \mathbb{Z}}$ for all $\alpha \in I_{r}$ and all $j, k \in \mathbb{Z}$.

Proof. The statement is clear from the above discussion for $k \geqslant \alpha+1$ for which all the partition functions in the determinant (5.14) have the form $z_{t, u}^{0,0}$ with $t \leqslant u$, and therefore can be interpreted within the LGV framework. For $k \geqslant 0$ however, from the structure of the $T$-system, it is clear that $T_{\alpha, j, k}$ only depends on a finite part of the initial data $\left\{T_{\beta, \ell, 0}, T_{\beta, \ell, 1},|\beta-\alpha|<k,|\ell-j|<k\right\}$. In particular, if $0<k<\alpha+1$, then as only the $\beta \geqslant \alpha+1-k>0$ are involved, we may truncate the size of the $T$-system to some $A_{r^{\prime}}$, with $r^{\prime}=r-(\alpha+1-k)<r$. Upon renaming the initial data accordingly, we may interpret $T_{\alpha, j, k}$ as $T_{\alpha^{\prime}, j, k}$ in this new $T$-system, where $\alpha^{\prime}=\alpha-(\alpha+1-k)=k-1$. For this $\alpha^{\prime} \geqslant k-1$ the LGV formula applies, and positivity follows. Finally, note that the

expression of $T_{\alpha, j, 1-k}$ in terms of $\left\{T_{\beta, j, 0}, T_{\beta, j, 1}\right\}$ is the same as that of $T_{\alpha, j, k}$ in terms of the reflected initial data $\left\{T_{\beta, j, 1}, T_{\beta, j, 0}\right\}$, hence positivity follows for $k<0$ as well.

\section{Operator formulation and positivity in terms of mutated initial data}

Let $\mathcal{A}$ be the space of Laurent polynomials in the variables $\left\{T_{\alpha, j, k}\right\}$. We consider the invertible "shift operator" $d$ acting on the infinite-dimensional vector space over $\mathcal{A}$ with basis $\{|t\rangle: t \in \mathbb{Z}\}$, with $d|t\rangle=|t-1\rangle$. It acts on the restricted dual space $V^{*}$, with basis $\langle t|$ such that $\left\langle t \mid t^{\prime}\right\rangle=\delta_{t, t^{\prime}}$, as $\langle t| d=\langle t+1|$. We consider the algebra of formal Laurent series in $d$ with coefficients in $\mathcal{A}$ acting on $V$. All operator relations which we derive below are considered in the weak sense, as identities between matrix elements. We also adopt the operator notation for diagonal operators in this basis, for example, $w_{a, b}|t\rangle=w_{a, b}(t)|t\rangle$.

\subsection{An expression using operator continued fractions}

Theorem 5.5 implies

$$
T_{1, j, k}=T_{1, j+k, 0}(\mathcal{T}(j-k) \mathcal{T}(j-k+1) \cdots \mathcal{T}(j+k-1))_{0,0}
$$

where the transfer matrix $\mathcal{T}(s)$ is defined in Equation (5.6). 

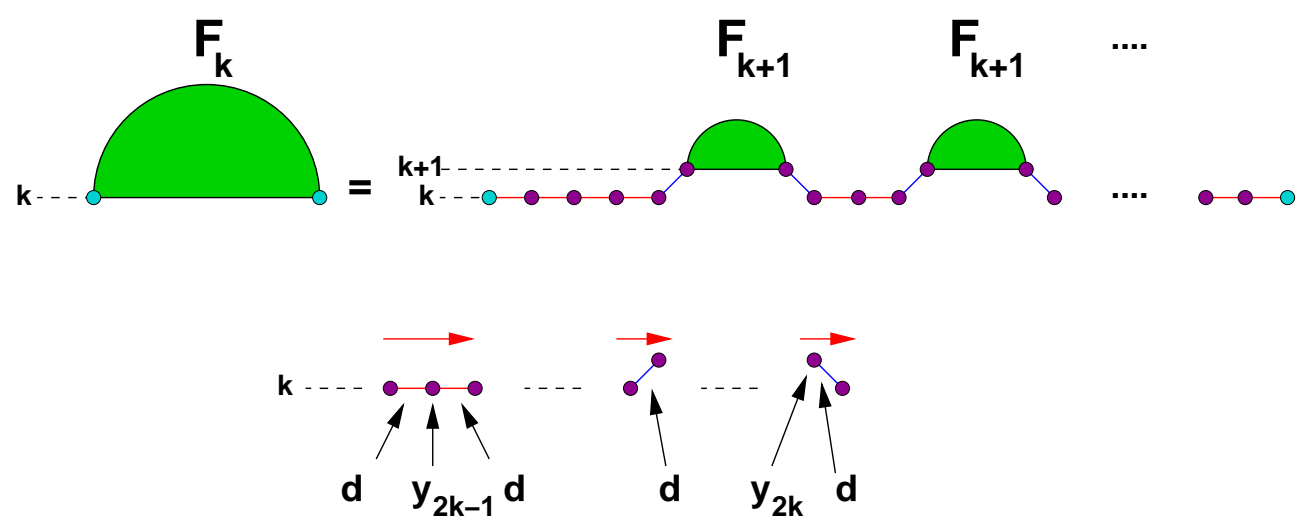

Figure 6.1: The enumeration of paths on $\widetilde{G}_{r}$ with time-dependent weights $y_{\alpha}(t)$. The paths from and to height $k$ which stay above height $k$ (generated by $\mathbb{F}_{k}$ ) are related to those from height $k+1$ by arranging any number of horizontal step-pairs $k \rightarrow k \rightarrow k$ and up-down step-pairs $k \rightarrow k+1 \rightarrow k$, in-between which we insert any path from and to height $k+1$ staying above height $k+1$. The operator weights are indicated on the bottom.

We define the operator-valued transfer matrix $\mathbb{T}$ to be the matrix with entries $\langle t| \mathbb{T}_{a, b}=$ $\mathcal{T}_{a, b}(t)\langle t+1|$. We also define operator-valued weights $\mathbb{Y}_{\alpha}$, such that

$$
\langle t| \mathbb{Y}_{\alpha}=y_{\alpha}(t, 0)\langle t+1|
$$

where the $y$ 's are defined in (4.13).

Using these, we can write

$$
T_{1, j, k}=T_{1, j+k, 0}\left\langle j-k\left|\left(\mathbb{T}^{2 k}\right)_{0,0}\right| j+k\right\rangle=T_{1, j+k, 0}\left\langle j-k\left|\left((I-\mathbb{T})^{-1}\right)_{0,0}\right| j+k\right\rangle,
$$

where $(I-\mathbb{T})^{-1}:=\sum_{n \geqslant 0}(\mathbb{T})^{n}$. Therefore, the operator $\mathbb{F}=\left((I-\mathbb{T})^{-1}\right)_{0,0}$ generates the variables $T_{1, j, k}$.

To compute $\mathbb{F}$, we row-reduce the matrix $I-\mathbb{T}$. The result can be written as a non-commutative continued fraction:

$$
\mathbb{F}=\left(1-d\left(1-d\left(1-d \mathbb{Y}_{3}-d\left(\ldots\left(1-d \mathbb{Y}_{2 r-1}-d\left(1-d \mathbb{Y}_{2 r+1}\right)^{-1} \mathbb{Y}_{2 r}\right)^{-1} \ldots\right)^{-1} \mathbb{Y}_{4}\right)^{-1} \mathbb{Y}_{2}\right)^{-1} \mathbb{Y}_{1}\right)^{-1}
$$

Alternatively, we can write $\mathbb{F}=\mathbb{F}_{0}$ where the operators $\mathbb{F}_{i}$ are defined inductively:

$$
\begin{aligned}
\mathbb{F}_{r+2} & =0, \\
\mathbb{F}_{k} & =\left(1-d \mathbb{Y}_{2 k-1}-d \mathbb{F}_{k+1} \mathbb{Y}_{2 k}\right)^{-1}, \quad(k=r+1, r, \ldots, 3,2), \\
\mathbb{F}_{1} & =\left(1-d \mathbb{F}_{2} \mathbb{Y}_{2}\right)^{-1}, \quad \mathbb{F}_{0}=\left(1-d \mathbb{F}_{1} \mathbb{Y}_{1}\right)^{-1}=\mathbb{F},
\end{aligned}
$$

where each term is understood as a formal power series in $d$.

This expression is easily understood in terms of paths. Note that each time increment corresponds to an insertion of an operator $d$. An up step at height $k$ followed by a down 
step contributes a weight $d \mathbb{Y}_{2 k}$, while a level step at height $k$ contributes the weight $d \mathbb{Y}_{2 k-1}$.

The operator generating function for paths above height $k, \mathbb{F}_{k}$, is obtained by shuffling the two following possibilities: (i) a level step pair $k \rightarrow k \rightarrow k$ (ii) insertion of a path above height $k+1$ between steps $k \rightarrow k+1$ and $k+1 \rightarrow k$ (see Figure 6.1 ).

\subsection{Mutations and operator continued fractions}

As with the $Q$-system of [6], we would like to have expressions for $T_{\alpha, j, k}$ as functions of other possible initial data of cluster seeds in the cluster algebra. Cluster positivity means that they are positive Laurent polynomials in this data, and we can prove this by giving path generating functions on graphs with positive weights for them. The operator formulation introduced above was designed to allow us to do this in the case of special seeds of the form

$$
\mathbf{x}_{\mathbf{M}}=\left\{T_{\alpha, j, m_{\alpha}+i} \mid i=0,1, \alpha \in I_{r}, j \in \mathbb{Z}\right\},
$$

where $\mathbf{M}$ is a Motzkin path of length $r: \mathbf{M}=\left(m_{1}, \ldots, m_{r}\right)$ with $\left|m_{i}-m_{i+1}\right| \leqslant 1$.

This case is special, because it is the non-commutative version of our construction in [6] for the $Q$-system. The only difference is that we must now use the operator-valued transfer matrix, instead of a scalar, to account for time-dependent weights.

\subsubsection{Compound mutations and restricted initial data}

The cluster seeds in Equation (6.5) are obtained from $\mathbf{x}_{0}$ by acting on it with a sequence of the compound mutations of the form

$$
\mu_{\alpha}=\prod_{j \in \mathbb{Z}} \mu_{\alpha, j}, \quad \mu_{\bar{\alpha}}=\prod_{j \in \mathbb{Z}} \mu_{\bar{\alpha}, j} .
$$

Note that the mutation matrix $B_{0}$ has the property that $B_{\alpha, \alpha}^{j, j^{\prime}}=0$ if $j \neq j^{\prime}$, hence $\mu_{\alpha, j}$ commutes with $\mu_{\alpha, j^{\prime}}$, so the compound mutations are well-defined.

The mutations (6.6) act on initial data $\mathbf{x}_{0}$ via the simultaneous use of all relations (1.1) for all $j \in \mathbb{Z}$ to transform $T_{\alpha, j ; k-1} \rightarrow T_{\alpha, j ; k+1}$ (forward mutation) or $T_{\alpha, j ; k+1} \rightarrow$ $T_{\alpha, j ; k-1}$ (backward mutation), the action being that of $\mu_{\alpha}$ when $k$ is odd and $\mu_{\bar{\alpha}}$ when $k$ is even. Starting from the seed $\mathbf{x}_{0}$, and acting only with (6.6) generates a restricted set of cluster seeds. If, moreover, we require that each of the mutations be one of the $T$-system equations, we obtain only seeds of the form $\mathbf{x}_{\mathbf{M}}$ as in (6.5).

Remark 6.1. This is very similar to the situation of [6], where seeds of type $\mathbf{x}_{\mathbf{M}}$ consist of variables $\left\{R_{\alpha, m_{\alpha}} ; R_{\alpha, m_{\alpha}+1}\right\}$. Here, we replace each variable $R_{\alpha, m}$ with the infinite sequence $\left(T_{\alpha, j, m}\right)_{j \in \mathbb{Z}}$.

\subsubsection{Operator continued fraction rearrangements}

In [6], we have shown that the generating function for $R_{1, n}$ may be expressed in terms of any mutated seed $\mathbf{x}_{\mathbf{M}}$ via local rearrangements of the initial continued fraction in terms 
of the seed $\mathbf{x}_{0}$. Here, we give the non-commutative version of the starting point, which is operator version of the two rearrangement lemmas for fractions used in [6].

The following are operator identities to be understood as identities between matrix elements of Laurent series in $d$. They are proved by a simple calculation.

Lemma 6.2. Let $\mathbb{A}, \mathbb{B}$ be elements in $\mathcal{A}((d))$. Then

$$
1+d(1-\mathbb{A} d-d \mathbb{B})^{-1} \mathbb{A}=\left(1-d(1-d \mathbb{B})^{-1} \mathbb{A}\right)^{-1}
$$

Lemma 6.3. Let $\mathbb{A}, \mathbb{B}, \mathbb{C}, \mathbb{U}$ be elements in $\mathcal{A}((d))$, with $\mathbb{A}+\mathbb{B}$ invertible. Then

$$
\mathbb{A}+\left(1-d(1-\mathbb{U})^{-1} \mathbb{C}\right)^{-1} \mathbb{B}=\left(1-\mathbb{U}-d\left(1-d \mathbb{C}^{\prime}\right)^{-1} \mathbb{B}^{\prime}\right)^{-1} \mathbb{A}^{\prime}
$$

where

$$
\mathbb{A}^{\prime}=\mathbb{A}+\mathbb{B}, \quad \mathbb{B}^{\prime}=\mathbb{C} \mathbb{B}(\mathbb{A}+\mathbb{B})^{-1}, \quad \mathbb{C}^{\prime}=d^{-1} \mathbb{C} \mathbb{A}(\mathbb{A}+\mathbb{B})^{-1} d
$$

Remark 6.4. Lemma 6.2 has a path interpretation. The r.h.s. of equation (6.7) is the generating function for paths on the integer segment $[0,2]$, from vertex 0 to 0 , with operator-valued weights:

$$
w(0 \rightarrow 1)=w(1 \rightarrow 2)=d, \quad w(2 \rightarrow 1)=\mathbb{B}, \quad w(1 \rightarrow 0)=\mathbb{A}
$$

The l.h.s. of equation (6.7) decomposes these paths into the trivial one (length 0, contribution 1), and all the others, which start with a step $0 \rightarrow 1$ and end up with a step $1 \rightarrow 0$, with respective weights $d$ and $\mathbb{A}$ (in this order). In-between, we have the generating function for "rerooted" paths, from vertex 1 to vertex 1 , which consist of arbitrary sequences of either steps $1 \rightarrow 0 \rightarrow 1$ (with weight $\mathbb{A} d$ ) or steps $1 \rightarrow 2 \rightarrow 1$ (with weight $d \mathbb{B}$ ). We call the rearrangement of this Lemma a "rerooting".

\subsubsection{General case: mutations as rearrangements}

For each Motzkin path $\mathbf{M}$, the solution of the $T$-system is can be expressed in terms of the initial data at $\mathbf{x}_{\mathbf{M}}$ as

$$
T_{1, j, k}=T_{1, j+k-m_{1}, m_{1}}\left\langle j-k+m_{1}\left|\mathbb{F}_{\mathbf{M}}\right| j+k-m_{1}\right\rangle
$$

for some operator continued fraction $\mathbb{F}_{\mathbf{M}}$. Our main claim is that this fraction is obtained from $\mathbb{F}(6.4)$ via a succesion of applications of Lemmas 6.2 and 6.3.

For each Motzkin path we will define weights $\mathbb{Y}_{i}(\mathbf{M}),\left(i \in I_{2 r+1}\right)$, which are monomials in $\mathbf{x}_{\mathbf{M}}$ and $d$. The fraction $\mathbb{F}_{\mathbf{M}}$ is a function of these. As in [6], we find that the effect of mutations on $\mathbb{F}_{\mathbf{M}} \mapsto \mathbb{F}_{\mathbf{M}^{\prime}}$ is the following:

- If $\alpha=1$, use Lemma 6.2 to write

$$
\mathbb{F}_{\mathbf{M}}=1+d \mathbb{F}_{\mathbf{M}}^{\prime} \mathbb{Y}_{1}(\mathbf{M}) .
$$

where $\langle t| \mathbb{Y}_{1}(\mathbf{m})=T_{1, t, m_{1}+1} / T_{1, t+1, m_{1}}\langle t+1|$. Then Lemma 6.3 enables us to rewrite $\mathbb{F}_{\mathbf{M}}^{\prime}$ as $\mathbb{F}_{\mathbf{M}^{\prime}}$, a function of $\mathbf{x}_{\mathbf{M}^{\prime}}$. 
- If $\alpha>1$, apply Lemma 6.3 to the part of $F_{\mathbf{M}}$ involving the weights $\mathbb{Y}_{\beta}(\mathbf{M})$ with $\beta \geqslant 2 \alpha-1$.

In both cases, the weights $\mathbb{Y}_{\beta}(\mathbf{M})$ are transformed into weights $\mathbb{Y}_{\beta}\left(\mathbf{M}^{\prime}\right)$.

We will provide the precise construction and proof in Section 6.3, but for clarity, we refer the reader to Appendix $\mathrm{B}$, where the example of $A_{2}$ is worked out completely.

\subsection{Paths on graphs with non-commutative weights}

In this section, we define graphs with weights in $\mathcal{A}\left[d, d^{-1}\right]$. The generating functions $\mathbb{F}_{\mathbf{M}}$ are path partition functions on these graphs. The graphs are identical to those introduced in [6], and the weights contain exactly the same information contained in the two-dimensional representation of paths on these graphs introduced in [6]. The construction presented here is therefore a rephrasing of these paths in terms of operators.

Remark 6.5. Although the two-dimensional representation of paths used here is identical to the one we used in [6], we did not, in the earlier paper, have use for the full information contained in this path representation. In particular, the horizontal coordinate ("time" in our language) had no interpretation in the context of $Q$-systems. Here, it corresponds to what is known as the spectral parameter in the T-system equations.

Since one is used to reading lattice paths from left to right, we have chosen to act on the space $V^{*}$ instead of $V$. In that way, the order in which they act on the space is the same as the order the path is traversed.

\subsection{The target graphs $\Gamma_{\mathrm{m}}$}

Let $\mathbf{M}$ be a Motzkin path. We decompose it into pieces which do not change direction: $\mathbf{M}=\mathbf{M}_{1} \cup \mathbf{M}_{2} \cup \cdots$, where $\mathbf{M}_{i}=\left(a, a+k, a+2 k, \cdots, a+\left(l_{i}-1\right) k\right)$ with $k=0,1$ or -1 . The type of subpath is called $k$. All the graphs used below must be drawn vertically (see Fig.6.2), which makes unambiguous the notion of top and bottom edges.

We construct a graph $\Gamma_{\mathbf{M}_{i}}$ for each $i$ as follows:

- If $k=0$ then $\Gamma_{\mathbf{M}_{i}}=\widetilde{G}_{l_{i}}^{\prime \prime}$, the graph $\widetilde{G}_{l_{i}}$ of Figure 5.1 (represented vertically), with its bottom and top edges removed.

- If $k=1$, then $\Gamma_{\mathbf{M}_{i}}$ is a simple (vertical) chain with $2 l_{i}$ vertices.

- If $k=-1$, then $\Gamma_{\mathbf{M}_{i}}$ is the graph $\widetilde{G}_{l_{i}}^{\prime \prime}$ (represented vertically) decorated with additional oriented "descending" edges $b \rightarrow a$ with $l_{i}+1 \geqslant b>a+1>1$.

We then glue the graphs: $\Gamma_{\mathbf{M}_{i}} \mid \Gamma_{\mathbf{M}_{i+1}}$ is the graph obtained by identifying the top edge of $\Gamma_{\mathbf{M}_{i}}$ with the bottom edge of $\Gamma_{\mathbf{M}_{i+1}}$. Define $\Gamma_{\mathbf{M}}^{\prime \prime}=\Gamma_{\mathbf{M}_{1}}\left|\Gamma_{\mathbf{M}_{2}}\right| \cdots$, and $\Gamma_{\mathbf{M}}$ is $\Gamma_{\mathbf{M}}^{\prime \prime}$ together with one additional bottom and top edge and vertex. The graph $\Gamma_{\mathbf{M}}$ is rooted at its bottom vertex. 


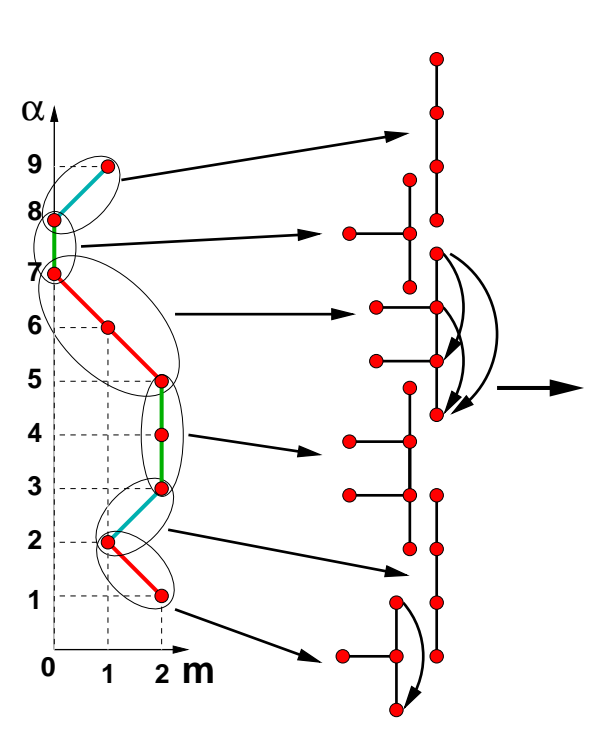

(a)

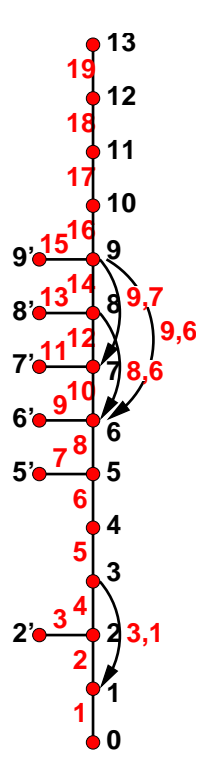

(c)

Figure 6.2: The construction of the graph $\Gamma_{\mathbf{M}}$ (c) for some sample Motzkin path $\mathbf{M}$ (a) for $A_{9}$. All the pieces glued (b) are represented vertically. The skeleton edges in (c) are labelled 1 to 19 , the spine vertices 0 to 13 .

Each graph thus constructed has a spine, namely a maximal vertical chain of vertices consisting of the unprimed vertices of the various pieces glued. We label the spine vertices consecutively starting from 0 at the bottom (see Fig.6.2(c)). The vertices off the spine, which are attached only to a vertex $i$ are labeled $i^{\prime}$. We define the skeleton of $\Gamma_{\mathbf{M}}$ as the graph with all edges $i \rightarrow j$ removed where $i>j+1$. The edges of the skeleton, referred to as skeleton edges are labeled $1,2, \ldots, 2 r+1$ from bottom to top (see Fig.6.2(c)).

A path traverses each edge of a graph in one direction or another, and in our formulation, we weight steps in each direction differently. Therefore, we now consider the non-oriented edges in $\Gamma_{\mathbf{M}}$ as doubly-oriented edges, each orientation corresponding to a different weight.

Assign a weight $\mathbb{Y}_{a, b}(\mathbf{M})$ to the edge $a \rightarrow b$. The weight of any edge away from the root, $\mathbb{Y}_{i, i+1}=\mathbb{Y}_{j, j^{\prime}}=d$. Skeleton edges $\mathbb{Y}_{\alpha}(\mathbf{M})$ pointing towards the root are independent weights, which we define below. Weights on edges $i \rightarrow i-k$ with $k>1$ are defined as the following product involving only skeleton weights or their inverses:

$$
\mathbb{Y}_{i, i-k}=\left(\prod_{a=i-1}^{i-k+1} \mathbb{Y}_{a+1, a}\left(\mathbb{Y}_{a, a^{\prime}}\right)^{-1}\left(\mathbb{Y}_{a^{\prime}, a}\right)^{-1}\right) \mathbb{Y}_{i-k+1, i-k}
$$

The ordered product is taken over edges from top to bottom, along a path from vertex $i$ to $i-k$. Note that $\mathbb{Y}_{a, a^{\prime}}=d$. By inspection of (6.12) we see that $\langle t| \mathbb{Y}_{i, i-k} \propto\langle t-k+2|$, hence $\mathbb{Y}_{i, i-k}$ "goes back in time" by $k-2$ units. 


\subsection{The positivity theorems for $\left\{T_{\alpha, j, k}\right\}$}

We now write $T_{1, j, k}$ as the partition function for paths on $\Gamma_{\mathbf{M}}$. The values of the skeleton weights are determined by considering the effect of a mutation on the seed data - They are determined by a recursion relation, which can be solved explicitly.

\subsubsection{Transfer matrices and mutations}

As we illustrated in [6], any Motzkin path has a unique expression as a sequence of forward mutations, $m_{\beta} \mapsto m_{\beta}^{\prime}=m_{\beta}+\delta_{\beta, \alpha}$ where $\mathbf{M}=\left(m_{1}, \ldots, m_{r}\right)$ and $\mathbf{M}^{\prime}=\left(m_{1}^{\prime}, \ldots, m_{r}^{\prime}\right)$. We restrict the mutations to those which increase $m_{\alpha}$ by +1 only in the following two cases:

- Case (i): $m_{\alpha-1}=m_{\alpha}=m_{\alpha+1}-1$,

- Case (ii): $m_{\alpha-1}=m_{\alpha}=m_{\alpha+1}$,

(together with their boundary versions). This restricted set of mutations is sufficient to construct all Motzkin paths in the fundamental domain.

The initial step in the induction is the Motzkin path $\mathbf{M}_{0}$. The path interpretation on $\Gamma_{\mathbf{M}_{0}}=\widetilde{G}_{r}$ was given in Section 5. The operator transfer matrix $\mathbb{T}_{\mathbf{M}_{0}}=\mathbb{T}$ and the operator generating function $\mathbb{F}_{\mathbf{M}_{0}}=\mathbb{F}$ are expressed entirely in terms of the $d$ operator and the skeleton weights $\mathbb{Y}_{\alpha}\left(\mathbf{M}_{0}\right)=\mathbb{Y}_{\alpha}(6.2)$.

The inductive step is as follows. Given $\Gamma_{\mathbf{M}}$ and its operator weights, consider a forward mutation $\mu_{\alpha}$ or $\mu_{\bar{\alpha}}: \mathbf{M} \mapsto \mathbf{M}^{\prime}$. These have associated transfer matrices $\mathbb{T}_{\mathbf{M}}$ and $\mathbb{T}_{\mathbf{M}^{\prime}}$ corresponding to the graphs $\Gamma_{\mathbf{M}}$ and $\Gamma_{\mathbf{M}^{\prime}}$. We compare the associated generating functions $\mathbb{F}_{\mathbf{M}}=\left(I-\mathbb{T}_{\mathbf{M}}\right)_{0,0}^{-1}$ and $\mathbb{F}_{\mathbf{M}^{\prime}}=\left(I-\mathbb{T}_{\mathbf{M}^{\prime}}\right)_{0,0}^{-1}$ using the row reduction process. Both are operator continued fractions, which differ locally due to the struction of the graphs. We find that the two operator continued fractions are equal to each other if and only if the weights of the graph $\Gamma_{\mathbf{M}^{\prime}}$ are related to those of $\Gamma_{\mathbf{M}}$ as follows.

Theorem 6.6. Let $\mathbb{Y}^{\prime}=\mathbb{Y}\left(\mathbf{M}^{\prime}\right)$ and $\mathbb{Y}=\mathbb{Y}(\mathbf{M})$, where $\mathbf{M}^{\prime}=\mu_{\alpha}(\mathbf{M})$ or $\mu_{\bar{\alpha}}(\mathbf{M})$. If $\alpha \neq 1$, then,

- Case (i):

$$
\begin{aligned}
\mathbb{Y}_{2 \alpha-1}^{\prime} & =\mathbb{Y}_{2 \alpha-1}+\mathbb{Y}_{2 \alpha} \\
\mathbb{Y}_{2 \alpha}^{\prime} & =\mathbb{Y}_{2 \alpha+1} \mathbb{Y}_{2 \alpha}\left(\mathbb{Y}_{2 \alpha-1}^{\prime}\right)^{-1} \\
\mathbb{Y}_{2 \alpha+1}^{\prime} & =d^{-1} \mathbb{Y}_{2 \alpha+1} \mathbb{Y}_{2 \alpha-1}\left(\mathbb{Y}_{2 \alpha-1}^{\prime}\right)^{-1} d
\end{aligned}
$$

- Case (ii): in addition to the previous, we have

$$
\mathbb{Y}_{2 \alpha+2}^{\prime}=d^{-1} \mathbb{Y}_{2 \alpha+2} \mathbb{Y}_{2 \alpha-1}\left(\mathbb{Y}_{2 \alpha-1}^{\prime}\right)^{-1} d, \quad \text { and } \quad \mathbb{Y}_{\beta}^{\prime}=d^{-1} \mathbb{Y}_{\beta} d, \quad \forall \beta \geqslant 2 \alpha+3
$$

If $\alpha=1$, we simply have to substitute $\mathbb{Y}_{1} \rightarrow d^{-1} \mathbb{Y}_{1} d$ in the above formulas. 
Proof. The proof is by Gaussian elimination as in [6]. The case $\alpha=1$ is special, as it requires a rerooting of the generating function. The transformation of weights must be applied on $\mathbb{F}_{\mathbf{M}}^{\prime}=\left(I-\mathbb{T}_{\mathbf{M}}\right)_{1,1}^{-1}$ as in (6.11), which induces the substitution $\mathbb{Y}_{1} \rightarrow d^{-1} \mathbb{Y}_{1} d$.

Let

$$
\lambda_{\alpha, t, m}=\frac{T_{\alpha, t, m+1}}{T_{\alpha, t+1, m}}, \quad \mu_{\alpha, t, m}=\frac{T_{\alpha, t, m}}{T_{\alpha-1, t+1, m}}
$$

Corollary 6.7. The skeleton weights obeying the recursions of Theorem 6.6, subject to the initial condition (6.2) are the operators $\mathbb{Y}_{\beta}(\mathbf{M})$, acting as $\langle t| \mathbb{Y}_{\beta}(\mathbf{M})=y_{\beta}(\mathbf{M} ; t)\langle t+1|$, with:

$$
\begin{aligned}
y_{2 \alpha-1}(\mathbf{M} ; t)= & \frac{\lambda_{\alpha, t+m_{\alpha}-m_{1}-1, m_{\alpha}}}{\lambda_{\alpha-1, t+m_{\alpha-1}-m_{1}, m_{\alpha-1}}} \\
y_{2 \alpha}(\mathbf{M} ; t)= & \frac{\mu_{\alpha+1, t+m_{\alpha}-m_{1}, m_{\alpha}+1}}{\mu_{\alpha, t+m_{\alpha}-m_{1}, m_{\alpha}}} \times\left\{\begin{array}{cc}
\frac{\lambda_{\alpha+1, t+m_{\alpha+1}-m_{1}, m_{\alpha+1}}}{\lambda_{\alpha+1, t+m_{\alpha}-m_{1}, m_{\alpha}}} & \text { if } m_{\alpha}=m_{\alpha+1}+1 \\
1 & \text { otherwise }
\end{array}\right\} \\
& \times\left\{\begin{array}{cc}
\frac{\lambda_{\alpha-1, t+m_{\alpha}-m_{1}, m_{\alpha}}}{\lambda_{\alpha-1, t+m_{\alpha-1}-m_{1}, m_{\alpha-1}}} & \text { if } m_{\alpha}=m_{\alpha-1}-1 \\
1 & \text { otherwise }
\end{array}\right\}
\end{aligned}
$$

Proof. By direct check of the recursion relations (6.13-6.14).

Thus, we have two expressions for the generating function of $T_{1, j, k}$, one in terms of the seed data $\mathbf{x}_{\mathbf{M}}$ and the other in terms of the seed data $\mathbf{x}_{\mathbf{M}^{\prime}}$. We call the transition between the two expressions a mutation: It acts on the graph $\Gamma_{\mathbf{M}}$ and on its weights. Alternatively, it acts on the operator continued fraction expresson for $\mathbb{F}_{\mathbf{M}}$ as a rearrangement.

\subsubsection{Positivity of $T_{1, j, k}$}

We note that the weights $(6.16) y_{\alpha}(\mathbf{M} ; t)$ are positive Laurent monomials of the initial data at $\mathbf{x}_{\mathbf{M}}$. We therefore have a positivity result:

Theorem 6.8. $T_{1, j, k+m_{1}} / T_{1, j+k, m_{1}}$ is the partition function for paths on the rooted graph $\Gamma_{\mathbf{M}}$ with the weights of Theorem 6.7, starting from the root at time $j-k$ and ending at the root at time $j+k$. As such it is a positive Laurent polynomial of the mutated data at $\mathbf{x}_{\mathbf{M}}$.

\subsubsection{General solution and strongly non-intersecting paths}

We now turn to the expression of $T_{\alpha, j, k}$ in terms of the mutated initial data $\mathbf{x}_{\mathbf{M}}$. We will interpret the determinant formula Theorem 3.1 for $T_{\alpha, j, k}$ à la Gessel-Viennot, in terms of the strongly non-intersecting paths on the graph $\Gamma_{\mathbf{M}}$ introduced in [6].

Let us briefly recall the two-dimensional $\Gamma_{\mathbf{M}}$-lattice paths used to represent paths on $\Gamma_{\mathbf{M}}$, given in [6]. There are fundamentally three kinds of oriented edges in $\Gamma_{\mathbf{M}}$ : the horizontal and vertical "skeleton" edges, and down-pointing long edges, with weights which depend on the skeleton weights. The steps taken along these edges on $\Gamma_{\mathbf{M}}$ are represented in $\mathbb{Z}^{2}$ as follows (see Fig.6.3 for an illustration): 

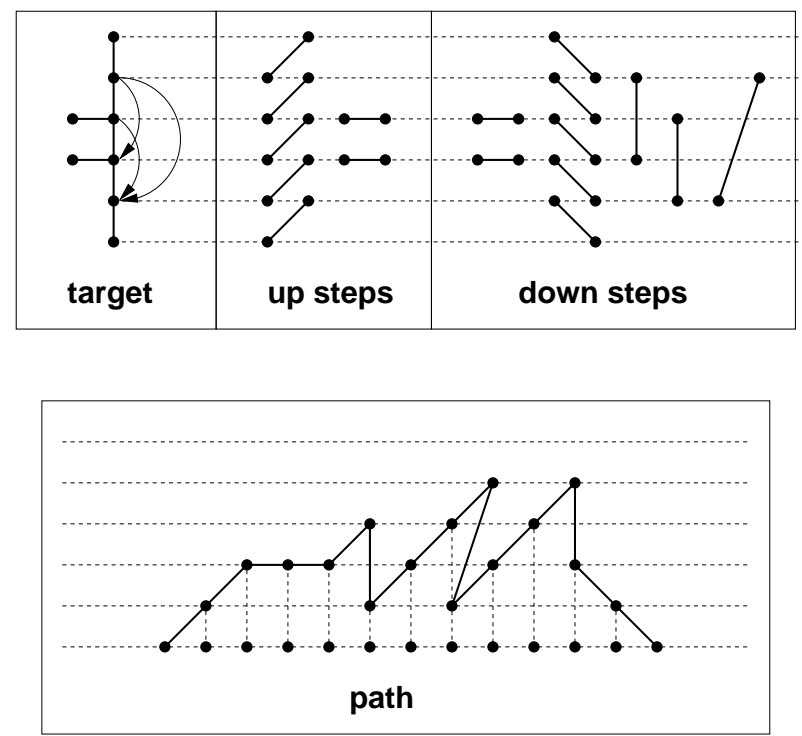

Figure 6.3: Two-dimensional lattice path representation on the graph $\Gamma_{\mathbf{M}}, \mathbf{M}=(2,1,0)$ of the $A_{3}$ case. We have indicated the "up" steps (i.e. away from the root) and the "down" steps (towards the root).

- A skeleton step $i \rightarrow i+\epsilon, \epsilon= \pm 1$, at time $t$ becomes the segment from $(t, i)$ to $(t+1, i+\epsilon)$

- A skeleton step $i \rightarrow i^{\prime}$ or $i^{\prime} \rightarrow i$ at time $t$ becomes the segment from $(t, i)$ to $(t+1, i)$

- A long step $j \rightarrow i, j>i+1$ at time $t$ becomes the segment from $(t, j)$ to $(t+j-i-2, i)$

Note that the increment of $x$-coordinate for each step coincides with the time shift we have associated with each step. Indeed, all steps advance by one unit of time, except the long ones, which go back in time by $j-i-2 \leqslant 0$. The only difference with [6] is that we now attach time-dependent weights to the steps namely a weight $y_{a, b}(t)$ for a step $a \rightarrow b$ starting at time $t$ (In the operator language, we have operator weights $\mathbb{Y}_{a, b}$ that act as $\langle t| \mathbb{Y}_{a, b}=y_{a, b}(t)\langle t+h|$, where $h$ is the time-shift of the corresponding step, $h=+1$ for all steps except the long ones, for which $h=a-b-2$.). We conclude that this representation is perfectly adapted to our weighted paths, as the $x$-coordinate is nothing but the time-coordinate.

Let us consider $T_{\alpha, j, k}$ as a function of the initial data at $\mathbf{x}_{\mathbf{M}}$. Writing

$$
\frac{T_{\alpha, j, k+m_{1}}}{\prod_{b=1}^{\alpha} T_{1, j+k+2 b-\alpha-1, m_{1}}}=\operatorname{det}_{1 \leqslant a, b \leqslant \alpha} \frac{T_{1, j-a+b, k+a+b-\alpha-1+m_{1}}}{T_{1, j+k+2 b-\alpha-1, m_{1}}}
$$

Using Theorem 6.8, we may interpret $T_{1, j-a+b, k+a+b-\alpha-1+m_{1}} / T_{1, j+k+2 b-\alpha-1, m_{1}}$ as the partition function for $\Gamma_{\mathbf{M}^{-}}$lattice paths from $s_{a}=(j-k+\alpha+1-2 a, 0)$ to $e_{b}=(j+k+2 b-\alpha-$ $1,0)$. The determinant is simply a signed sum of products of such path partition functions, corresponding in turn to the partition function for families of paths starting at $\left\{s_{a}\right\}_{a=1}^{\alpha}$ 


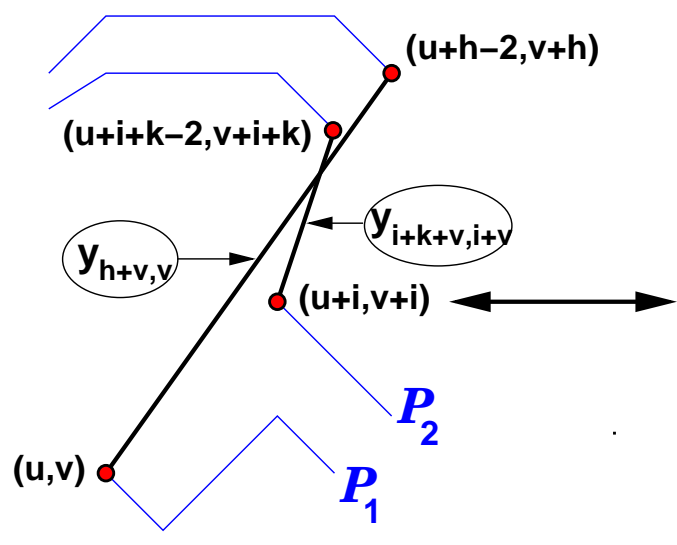

(a)

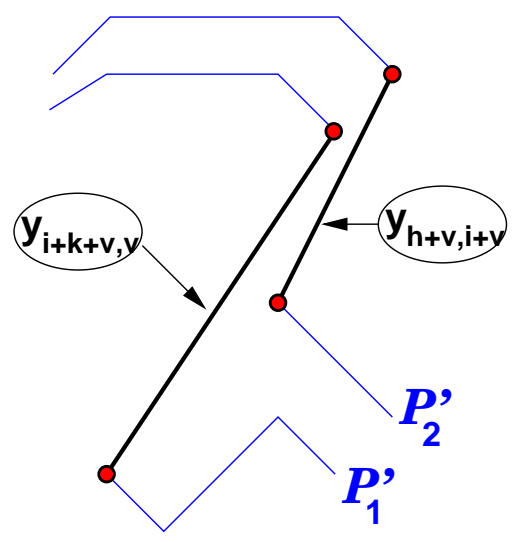

(b)

Figure 6.4: A typical edge intersection of $\Gamma_{\mathbf{M}}$-paths (a) and the result of the flipping operation on it (b). We have indicated the weights of the steps. The paths in (b) are said to be "too close" to each other.

and ending at $\left\{e_{b}\right\}_{b=1}^{\alpha}$, with the usual weights times the signature of the permutation of endpoints induced by the configuration.

In the standard Gessel-Viennot case, these signs produce the necessary cancellations to only leave us with the contribution of non-intersecting paths, namely families in which no two paths share a vertex. This is best proved by introducing a sign-reversing involution that pairs up and cancels all the unwanted terms in the expansion of the determinant.

In the case of $\Gamma_{\mathbf{M}}$-paths, the situation is more subtle, as paths may intersect without sharing a vertex. In [6], we have produced an involution, which allows to interpret an analogue of the determinant (6.19) as the partition function of strongly non-intersecting $\Gamma_{\mathbf{M}}$-lattice paths. This involution consists in flipping paths as follows. We consider the first intersection between two paths within a family. If the intersection is at a common vertex, we interchange the portions of paths before the intersection. If it is not at a common vertex, we flip the two paths as indicated in Fig.6.4, by switching their beginnings until the crossing.

We make then the following crucial observation:

Lemma 6.9. In the generic flipping situation of Fig.6.4, the flipped pair of paths has the same (time-dependent) weight as the original one, up to the sign of the permutation of starting points, due to the following relation:

$$
y_{h+v, v}(u+h-2) y_{i+k+v, i+v}(u+i+k-2)=y_{h+v, i+v}(u+h-2) y_{i+k+v, v}(u+i+k-2)
$$

Proof. By direct application of the formula (6.12) for the long edge weights.

The only invariant families under this involution are those where the paths do not lie "too close" to each-other, as otherwise they get cancelled by applying a flip.

Therefore all the conclusions of [6] still hold in the present case, and we have: 
Theorem 6.10. $T_{\alpha, j, k+m_{1}} / \prod_{b=1}^{\alpha} T_{\alpha, j+k+2 b-\alpha-1, m_{1}}$ is the partition function for configurations of $\alpha$ strongly non-intersecting $\Gamma_{\mathbf{M}}$-lattice paths, with the weights of Theorem 6.7. As such, $T_{\alpha, j, k+m_{1}}$ is a positive Laurent polynomial of the mutated data at $\mathbf{x}_{\mathbf{M}}$.

\section{Conclusion}

The $T$-system equations can be interpreted as a special case of a non-commutative $Q$ system. In other words, one can write a $Q$-system for non-commutative (operator) variables, whose matrix elements obey the $T$-system equations. One can think of the noncommutative $Q$-system as a "non-commutative" cluster algebra. Special cases of noncommutative cluster algebras have been considered in several contexts, for example the quantum cluster algebras of Berenstein and Zelevinsky [3], or the more general recursion relation introduced by Kontsevich [15] (in rank 2), with similar Laurent properties, and which can be solved in some special "affine" cases (i.e. related to affine Cartan matrices) using our methods [8]. The main idea is that the path formulation seems to be particularly well adapted to the explicit solution of such problems, and makes both Laurent and positivity properties of the solution as function of the initial data manifest. This will be discussed in a future publication.

We should mention that there has been a great deal of interest in $T$-systems with more restrictive boundary conditions $[21,13]$. We hope that the construction introduced in this paper will provide a simple way of treating such boundary conditions and their consequences.

\section{A Discrete Wronskians}

\section{A.1 Plücker relations}

Let $P$ be an $N \times(N+k)$-matrix. Let $\left|P^{b_{1}, \ldots, b_{k}}\right|$ be the determinant of the matrix obtained by deleting the $k$ columns $b_{1}, \ldots, b_{k}$ of $P$, times the signature of the permutation that reorders these column indices in increasing order. Then we have:

$$
\left|P^{a_{1}, \ldots, a_{k}}\right|\left|P^{b_{1}, \ldots, b_{k}}\right|=\sum_{p=1}^{k}\left|P^{b_{p}, a_{2} \ldots, a_{k}}\right|\left|P^{b_{1}, \ldots, b_{p-1}, a_{1}, b_{p+1}, \ldots, b_{k}}\right| .
$$

for any choice of $2 k$ columns $a_{1}, \ldots, a_{k}$ and $b_{1}, \ldots, b_{k}$ of $P$. In particular, when $k=2$, we have

$$
\left|P^{a_{1}, a_{2}}\right|\left|P^{b_{1}, b_{2}}\right|=\left|P^{b_{1}, a_{2}}\right|\left|P^{a_{1}, b_{2}}\right|+\left|P^{b_{2}, a_{2}}\right|\left|P^{b_{1}, a_{1}}\right| .
$$

for any $N \times(N+2)$ matrix $P$.

Equation (A.2) implies the Desnanot-Jacobi relation. Let $M$ be an $N \times N$ matrix, and let $|M|, M_{i}^{j},\left|M_{i_{1}, i_{2}}^{j_{1}, j_{2}}\right|$ denote the determinants of $M$, the minor obtained by erasing row $i$ and column $j$ of $M$, and the double minor obtained by erasing rows $i_{1}, i_{2}$ and columns 
$j_{1}, j_{2}$ of $M$, respectively. Let $1 \leqslant i_{1}<i_{2} \leqslant N$ and $1 \leqslant j_{1}<j_{2} \leqslant N$, then

$$
|M|\left|M_{i_{1}, i_{2}}^{j_{1}, j_{2}}\right|=\left|M_{i_{1}}^{j_{1}}\right|\left|M_{i_{2}}^{j_{2}}\right|-\left|M_{i_{1}}^{j_{2}}\right|\left|M_{i_{2}}^{j_{1}}\right|
$$

It is easily obtained as a particular case of eq.(A.2), for $a_{2}=j_{1}, b_{2}=j_{2}, P_{i, a_{1}}=\delta_{i, i_{1}}$, $P_{i, b_{1}}=\delta_{i, i_{2}}$, and $M$ is the matrix $P$ with columns $a_{1}$ and $b_{1}$ erased. Indeed, one checks directly that: $\left|P^{a_{1}, b_{1}}\right|=|M|,\left|P^{a_{2}, b_{2}}\right|=\left|M_{1, N}^{1, N}\right|,\left|P^{a_{1}, a_{2}}\right|=\left|M_{N}^{1}\right|,\left|P^{b_{2}, b_{1}}\right|=-\left|M_{1}^{N}\right|$, $\left|P^{a_{1}, b_{2}}\right|=\left|M_{N}^{N}\right|$, and $\left|P^{b_{1}, a_{2}}\right|=-\left|M_{1}^{1}\right|$.

\section{A.2 T-system as discrete Wronskians}

Here present the proof of Theorem 3.1 which uses the relations in the previous subsection.

Proof. Consider Equation (A.3). Let $N=\alpha+1, i_{1}=j_{1}=1, i_{2}=j_{2}=N$ and choose the matrix $M$ with entries $M_{a, b}=T_{1, j+a-b, k+a+b-\alpha-2}$ for $a, b=1,2, \ldots, \alpha+1$. We denote by $W_{\alpha+1, j, k}=|M|$ the corresponding "discrete Wronskian" determinant. Substituting this definition into eq. (A.3), we have

$$
W_{\alpha+1, j, k} W_{\alpha-1, j, k}=W_{\alpha, j, k-1} W_{\alpha, j, k+1}-W_{\alpha, j-1, k} W_{\alpha, j+1, k}
$$

valid for $\alpha \in I_{r}$, provided we set $W_{0, j, k}=1$. Note that $W_{1, j, k}=T_{1, j, k}$ by definition. Comparing eq.(A.4) with the $T$-system (1.1), we deduce that the $T$ 's and $W$ 's obey the same recursion relations and share the same initial conditions at $\alpha=0$ and 1 . As the system is a three-term recursion in $\alpha$ this determines the solution uniquely and therefore we have $W_{\alpha, j, k}=T_{\alpha, j, k}$ for all $\alpha \in I_{r}, j, k \in \mathbb{Z}$.

\section{A.3 Linear recursion relations}

Here, we present a proof of Theorem 3.2, that the variables $T_{\alpha, j ; k}$ satisfy linear recursion relations, with constant coefficients which are the conserved quantities.

Proof. We perform the discrete analog of differentiating the Wronskian, and compute $\varphi_{j-1, k+1}-\varphi_{j, k}=0$. Denoting by $\varphi_{j, k}=\left|\mathbf{g}_{1}, \mathbf{g}_{2}, \cdots, \mathbf{g}_{r+1}\right|$ and $\varphi_{j-1, k+1}=\left|\mathbf{f}_{1}, \mathbf{f}_{2}, \cdots, \mathbf{f}_{r+1}\right|$ as the determinants of column vectors $\mathbf{g}_{i}, \mathbf{f}_{i}$, we note that $\mathbf{g}_{i+1}=\mathbf{f}_{i}$ for $i=1,2, \ldots, r$. We may therefore rewrite

$$
\varphi_{j-1, k+1}-\varphi_{j, k}=0=\left|\mathbf{f}_{1}, \mathbf{f}_{2}, \cdots \mathbf{f}_{r}, \mathbf{f}_{r+1}-(-1)^{r} \mathbf{g}_{1}\right|
$$

with $\left(\mathbf{f}_{b}\right)_{a}=T_{1, j-1+a-b, k+a-r-1+b}$ and $\left(\mathbf{g}_{1}\right)_{a}=T_{1, j-1+a, k+a-r-1}$. Therefore, there must exist a non-trivial linear combination of the columns of the matrix which vanishes. We write it as

$$
\sum_{b=1}^{r}(-1)^{b} c_{r+1-b}(j, k) \mathbf{f}_{b}+c_{0}(j, k)\left(\mathbf{f}_{r+1}-(-1)^{r} \mathbf{g}_{1}\right)=0 .
$$

Recall that the entries of the vectors $\mathbf{f}_{b}$ depend on $j, k$ in a very particular way, namely $\left(\mathbf{f}_{b}(j, k)\right)_{a+1}=\left(\mathbf{f}_{b}(j+1, k+1)\right)_{a}$, and similarly for $\mathbf{g}_{1}$. In order for the above linear 
combination to be non-trivial, we must therefore have $c_{b}(j, k)=c_{b}(j-1, k-1)=\cdots=$ $c_{b}(j-k, 0)$ for all $j, k \in \mathbb{Z}$, hence the coefficients $c_{b}$ only depend on the difference $j-k$. Finally, we may normalize the coefficients in such a way that $c_{0}=1$ identically, and the first part of the Theorem follows.

The second part is treated analogously, by considering the difference of Wronskians $\varphi_{j+1, k+1}-\varphi_{j, k}=0$ and reasoning on the rows of the corresponding matrices.

\section{A.4 Conserved quantities as Wronskian determinants with de- fect}

Here, we give the proof of Lemma 3.4 expressing the conserved quantities of the $T$-system as Wronskian determinants with defects.

Proof. Let $\gamma_{m}(j, n)$ denote the right hand side of of eq.(3.5).

It is clear that that $\gamma_{0}(j, n)=T_{r+1, j+n, n+r}=1$ and $\gamma_{r+1}(j, n)=T_{r+1, j+n-1, n+r+1}=1$ as consequences of Theorem 3.1 and of the $A_{r}$ type boundary condition.

Let $p \in \mathbb{Z}$ and define the $(r+2) \times(r+2)$ matrix $D$ to be the matrix with entries $D_{1, b}=$ $T_{1, j+p+1-b, p+b-1}$, and $D_{a, b}=T_{1, j+n+a-b, n+a+b-2}$ for $a=2,3, \ldots, r+2$ and $b=1,2, \ldots, r+2$. The identity (3.3) may be recast into a vanishing non-trivial linear combination of the columns of $D$, with coefficients $c_{r+2-b}(j)(-1)^{b-1}, b=1,2, \ldots, r+2$, hence the determinant of $D$ vanishes.

Expanding the determinant along the first row, we find that

$$
0=\operatorname{det}(D)=\sum_{b=1}^{r+2}(-1)^{b+1} D_{1, b}\left|D_{1}^{b}\right|=\sum_{b=1}^{r+2}(-1)^{b-1} \gamma_{r+2-b}(j, n) T_{1, j+p+1-b, p+b-1},
$$

as the determinants $\gamma_{m}(j, n)$ are the minors $\left|P_{1}^{r+2-m}\right|$.

Since the Wronskian determinant $T_{r+1, j, k}=1$ is non-zero, there exist no other nontrivial linear recursion relation than Equation (3.3) with strictly fewer terms, hence the coefficients in Equation (A.7) must be proportional to those in Equation (3.3). As $c_{0}(j)=$ $\gamma_{0}(j, n)=1$, we deduce that $\gamma_{m}(j, n)=c_{m}(j)$ for all $m=0,1,2 \ldots, r+1$, and the Lemma follows.

\section{B Example of $A_{2}$ : rearrangements, graphs and paths}

Here, we illustrate the program of Section 6.2.3 in the case $r=2$. We first present the rearrangements of the operator continued fraction $F$, which make positivity of $R_{1, n}$ manifest in all three cases. Next, we interpret these in terms of partition functions for operator-weighted paths on graphs, to illustrate Section 6.3.

\section{B.0.1 Rearrangements}

The fundamental domain for the action of mutations on the fundamental seed $\widetilde{\mathbf{x}}_{0}$ is coded by the following three Motzkin paths with 2 vertices: $\mathbf{m}_{0}=(0,0), \mathbf{m}_{1}=\mu_{1}\left(\mathbf{m}_{0}\right)=$ 
$(1,0)$ and $\mathbf{m}_{2}=\mu_{2}\left(\mathbf{m}_{0}\right)=(0,1)$. We give below the three operator continued fractions corresponding to these points.

Seed $\mathbf{x}_{0}$ : The continued fraction $F_{0}(\mathbf{y})$ reads for the fundamental seed corresponding to the Motzkim path $\mathbf{m}_{0}$ is:

$$
F_{0}(\mathbf{y})=\left(1-d\left(1-d\left(1-d \mathbb{Y}_{3}-d\left(1-d \mathbb{Y}_{5}\right)^{-1} \mathbb{Y}_{4}\right)^{-1} \mathbb{Y}_{2}\right)^{-1} \mathbb{Y}_{1}\right)^{-1}
$$

with operators $\mathbb{Y}_{i}, i \in I_{5}$, acting as $\langle t| \mathbb{Y}_{i}=y_{i}(t)\langle t+1|$, and:

$$
\begin{aligned}
& y_{1}(t)=\frac{T_{1, t, 1}}{T_{1, t+1,0}}, \quad y_{2}(t)=\frac{T_{2, t, 1}}{T_{1, t, 0} T_{1, t+1,1}}, \quad y_{3}(t)=\frac{T_{1, t+1,0} T_{2, t-1,1}}{T_{1, t, 1} T_{2, t, 0}} \\
& y_{4}(t)=\frac{T_{1, t+1,0}}{T_{2, t, 0} T_{2, t+1,1}}, \quad y_{5}(t)=\frac{T_{2, t+1,0}}{T_{2, t, 1}}
\end{aligned}
$$

Seed $\mathbf{x}_{2}=\mu_{2}\left(\mathbf{x}_{0}\right)$ : Following Section 6.2 .3 , we apply the Lemma 6.3 to $F_{0}$, with $\mathbb{A}=\mathbb{Y}_{3}, \mathbb{B}=\mathbb{Y}_{4}, \mathbb{C}=\mathbb{Y}_{5}$ and $\mathbb{U}=0$ : This yields $F_{0}(\mathbf{y})=F_{2}(\mathbf{w})$, where

$$
F_{2}(\mathbf{w})=\left(1-d\left(1-d\left(1-d\left(1-d\left(1-d \mathbb{W}_{5}\right)^{-1} \mathbb{W}_{4}\right)^{-1} \mathbb{W}_{3}\right)^{-1} \mathbb{W}_{2}\right)^{-1} \mathbb{W}_{1}\right)^{-1}
$$

with operators $\mathbb{W}_{i}, i \in I_{5}$, acting as $\langle t| \mathbb{W}_{i}=w_{i}(t)\langle t+1|$, with:

$$
\begin{aligned}
& w_{1}(t)=\frac{T_{1, t, 1}}{T_{1, t+1,0}}, \quad w_{2}(t)=\frac{T_{2, t, 1}}{T_{1, t, 0} T_{1, t+1,1}}, \quad w_{3}(t)=\frac{T_{1, t+1,0} T_{2, t, 2}}{T_{1, t, 1} T_{2, t+1,1}} \\
& w_{4}(t)=\frac{T_{1, t+1,1}}{T_{2, t, 1} T_{2, t+1,2}}, \quad w_{5}(t)=\frac{T_{2, t+1,1}}{T_{2, t, 2}}
\end{aligned}
$$

To obtain this, we have written $\mathbb{W}_{1}=\mathbb{Y}_{1}, \mathbb{W}_{2}=\mathbb{Y}_{2}$, and $\mathbb{W}_{3}=\mathbb{Y}_{3}+\mathbb{Y}_{4}$, while $\mathbb{W}_{4}=$ $\mathbb{Y}_{5} \mathbb{Y}_{4} \mathbb{W}_{3}^{-1}$ and $\mathbb{W}_{5}=d^{-1} \mathbb{Y}_{5} \mathbb{Y}_{3} \mathbb{W}_{3}^{-1} d$, and used the $T$-system to simplify the expressions.

Seed $\mathbf{x}_{1}=\mu_{1}\left(\mathbf{x}_{0}\right)$ : Following Section 6.2.3, we first apply the rerooting Lemma 6.2, with $\mathbb{A}=\mathbb{Y}_{1}$ and $\mathbb{B}=\mathbb{V} \mathbb{Y}_{2}$, where

$$
\mathbb{V}=\left(1-d \mathbb{Y}_{3}-d\left(1-d \mathbb{Y}_{5}\right)^{-1} \mathbb{Y}_{4}\right)^{-1}
$$

This allows to rewrite $F_{0}(\mathbf{y})=1+d F_{0}^{\prime}(\mathbf{y}) \mathbb{Y}_{1}$, with $F_{0}^{\prime}(\mathbf{y})=\left(1-\mathbb{Y}_{1} d-d \mathbb{U Y}_{2}\right)^{-1}$. We may now apply the rearrangement Lemma 6.3 , with $\mathbb{A}=d^{-1} \mathbb{Y}_{1} d, \mathbb{B}=\mathbb{Y}_{2}, \mathbb{C}=\mathbb{Y}_{3}+(1-$ $\left.d \mathbb{Y}_{5}\right)^{-1} \mathbb{Y}_{4}$, and $\mathbb{U}=0$ : this yields $F_{0}(\mathbf{y})=1+d F_{1}(\mathbf{z}) \mathbb{Y}_{1}$, where:

$$
F_{1}(\mathbf{z})=\left(1-d\left(1-d\left(1-d \mathbb{Z}_{3}-d\left(1-d \mathbb{Z}_{5}\right)^{-1} \mathbb{Z}_{4}\right)^{-1}\left(\mathbb{Z}_{2}+\left(1-d \mathbb{Z}_{5}\right)^{-1} \mathbb{Z}_{6}\right)\right)^{-1} \mathbb{Z}_{1}\right)^{-1}
$$




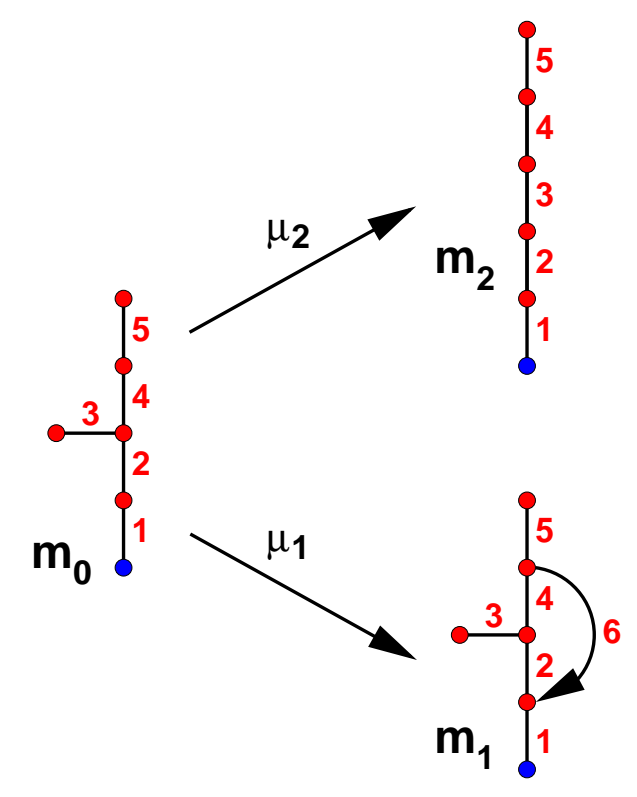

Figure B.1: The fundamental domain for $A_{2}$, coded by the Motzkin paths $\mathbf{m}_{0}, \mathbf{m}_{1}, \mathbf{m}_{2}$, and the corresponding target graphs for the path interpretation, with their edge labels. Mutations are indicated by arrows.

where $\mathbb{Z}_{i}, i \in I_{5}$ act as $\langle t| \mathbb{Z}_{i}=z_{i}(t)\langle t+1|$, with:

$$
\begin{aligned}
& z_{1}(t)=\frac{T_{1, t, 2}}{T_{1, t+1,1}}, \quad z_{2}(t)=\frac{T_{2, t-1,1} T_{2, t+1,1}}{T_{1, t, 1} T_{1, t+1,2} T_{2, t, 0}}, \quad z_{3}(t)=\frac{T_{1, t+1,1} T_{2, t-2,1}}{T_{1, t, 2} T_{2, t-1,0}} \\
& z_{4}(t)=\frac{T_{1, t-1,1} T_{1, t+1,1}}{T_{2, t-1,0} T_{2, t, 1} T_{1, t, 2}}, \quad z_{5}(t)=\frac{T_{2, t, 0}}{T_{2, t-1,1}}
\end{aligned}
$$

and $\mathbb{Z}_{6}$ is a long step weight, expressed in terms of the skeleton weights as: $\mathbb{Z}_{6}=$ $\mathbb{Z}_{4}\left(d \mathbb{Z}_{3}\right)^{-1} \mathbb{Z}_{2}$ (a particular case of Eq.(6.12)). Note that it is diagonal, namely:

$$
\langle t| z_{6}=z_{6}(t)\langle t|, \quad \text { where } \quad z_{6}(t)=\frac{z_{4}(t)}{z_{3}(t)} z_{2}(t-1)=\frac{1}{T_{1, t, 2} T_{2, t-1,0}}
$$

The above weights follow from the identifications: $\mathbb{Z}_{1}=d^{-1} \mathbb{Y}_{1} d+\mathbb{Y}_{2}, \mathbb{Z}_{2}=\mathbb{Y}_{3} \mathbb{Y}_{2} \mathbb{Z}_{1}^{-1}$, $\mathbb{Z}_{6}=d^{-1} \mathbb{Y}_{4} \mathbb{Y}_{2} \mathbb{Z}_{1}^{-1}, \mathbb{Z}_{3}=d^{-1} \mathbb{Y}_{3} d^{-1} \mathbb{Y}_{1} d \mathbb{Z}_{1}^{-1}, \mathbb{Z}_{4}=d^{-1} \mathbb{Y}_{4} d^{-1} \mathbb{Y}_{1} d \mathbb{Z}_{1}^{-1}$ and $\mathbb{Z}_{5}=d^{-1} \mathbb{Y}_{5} d$, and the use of the $T$-system to simplify the expressions.

\section{B.0.2 Paths on graphs with operator weights}

Recall first that the continued fractions $F_{0}(\mathbf{y}), F_{2}(\mathbf{w})$ are such that $T_{1, j, k} / T_{1, j+k, 0}=\langle j-$ $\left.k\left|F_{i}\right| j+k\right\rangle, i=0,2$, while, due to the re-rooting, we have $T_{1, j, k} / T_{1, j+k-1,1}=\langle j-k+$ $\left.1\left|F_{1}\right| j+k-1\right\rangle$.

The three above operator continued fractions may be interpreted in terms of path counting as follows. We have represented in Figure B.1 the three rooted target graphs 
$\Gamma_{\mathbf{m}}$, attached to the three Motzkin paths $\mathbf{m}=\mathbf{m}_{0}, \mathbf{m}_{1}, \mathbf{m}_{2}$, together with their edge labelings. We have the following

Theorem B.1. For $i=0,1,2$, the quantities $\left\langle t\left|F_{i}\right| t^{\prime}\right\rangle$ are the partition functions for paths on the graphs $\Gamma_{\mathbf{m}_{i}}$, from and to the root, starting at time $t$ and ending at time $t^{\prime}$, and with operator weights defined as the product over the operator weights for each successive step of the path, in the same order. The weights are $d$ per step away form the root, and respectively $\mathbb{Y}_{i}, \mathbb{Z}_{i}$ and $\mathbb{W}_{i}$ per step towards the root, along the edge labeled $i$.

Proof. The proof is a straightforward adaptation of the argument of Section 6.1: it uses operator transfer matrices $\mathbb{T}_{i}$, and amounts to performing the Gaussian elimination of $I-\mathbb{T}_{i}$, in order to compute $\left(\left(I-\mathbb{T}_{i}\right)^{-1}\right)_{0,0}$, where 0 indexes the root vertex on $\Gamma_{\mathbf{m}_{i}}$. We give explicit expressions below.

We now list the transfer matrices for the three cases above. In all cases, we have $F_{i}=\left(\left(I-\mathbb{T}_{i}\right)^{-1}\right)_{0,0}$.

$\mathbb{T}_{0}=\left(\begin{array}{cccccc}0 & d & 0 & 0 & 0 & 0 \\ \mathbb{Y}_{1} & 0 & d & 0 & 0 & 0 \\ 0 & \mathbb{Y}_{2} & 0 & d & 0 & 0 \\ 0 & 0 & \mathbb{Y}_{3} & 0 & d & d \\ 0 & 0 & \mathbb{Y}_{4} & 0 & 0 & 0 \\ 0 & 0 & 0 & 0 & \mathbb{Y}_{5} & 0\end{array}\right) \mathbb{T}_{1}=\left(\begin{array}{cccccc}0 & d & 0 & 0 & 0 & 0 \\ \mathbb{Z}_{1} & 0 & d & 0 & 0 & 0 \\ 0 & \mathbb{Z}_{2} & 0 & d & 0 & 0 \\ 0 & 0 & \mathbb{Z}_{3} & 0 & d & d \\ 0 & \mathbb{Z}_{6} & \mathbb{Z}_{4} & 0 & 0 & 0 \\ 0 & 0 & 0 & 0 & \mathbb{Z}_{5} & 0\end{array}\right) \mathbb{T}_{2}=\left(\begin{array}{cccccc}0 & d & 0 & 0 & 0 & 0 \\ \mathbb{W}_{1} & 0 & d & 0 & 0 & 0 \\ 0 & \mathbb{W}_{2} & 0 & d & 0 & 0 \\ 0 & 0 & \mathbb{W}_{3} & 0 & d & 0 \\ 0 & 0 & 0 & \mathbb{W}_{4} & 0 & d \\ 0 & 0 & 0 & 0 & \mathbb{W}_{5} & 0\end{array}\right)$

Remark B.2. Note that, as opposed to the two other cases, the transfer matrix $\mathbb{T}_{1}$ is not made of diagonal operators times $d$, as $\mathbb{Z}_{6}$ is diagonal, hence goes back one step in time compared to the other operators $\mathbb{Z}_{i}, i=1,2, \ldots, 5$. This necessity for the longer descending steps to go back in time was already observed in [6] in the two-dimensional representation of the $\Gamma_{\mathbf{m}}$-paths.

\section{References}

[1] V. Bazhanov and N. Reshetikhin, Restricted solid-on-solid models connected with simply-laced algebras and conformal field theory. J. Phys. A: Math. Gen. 23 (1990) 1477-1492.

[2] A. Berenstein, S. Fomin, A. Zelevinsky, Cluster algebras III: Upper bounds and double Bruhat cells Duke Math. J. 126 (2005), No. 1, 1-52.

[3] A. Berenstein, A. Zelevinsky, Quantum Cluster Algebras, Adv. Math. 195 (2005) 405-455. arXiv:math/0404446 [math.QA].

[4] P. Caldero and M. Reineke, On the quiver Grassmannian in the acyclic case J. Pure Appl. Algebra 212 (2008), 2369-2380. arXiv:math/0611074 [math.RT].

[5] P. Di Francesco and R. Kedem, Q-systems as cluster algebras II, Lett. Math. Phys. 89 No 3 (2009) 183-216, arXiv:0803.0362 [math.RT].

[6] P. Di Francesco and R. Kedem, Q-systems, heaps, paths and cluster positivity, Comm. Math. Phys. (2009) DOI 10.1007/s00220-009-0947-5 (76 pages). arXiv:0811.3027 [math.CO]. 
[7] P. Di Francesco and R. Kedem, Q-systems cluster algebras, paths and total positivity. Preprint arXiv:0906:3421 [math.CO].

[8] P. Di Francesco and R. Kedem, Discrete non-commutative integrability: the proof of a conjecture by $M$. Kontsevich, to appear.

[9] S. Fomin and A. Zelevinsky, Cluster algebras. I.Foundations, J. Amer. Math. Soc. 15 (2002), no. 2, 497-529.

[10] S. Fomin And A. Zelevinsky Double Bruhat cells and total positivity, Jour. of the A.M.S. 12, No 2 (1999), 335-380.

[11] E. Frenkel and N. Reshetikhin, The q-characters of representations of quantum affine algebras and deformations of $W$-algebras. In Recent developments in quantum affine algebras and related topics (Raleigh NC, 1998), Contemp. Math. 248 (1999), 163205.

[12] C. Geiss, B. Leclerc and J. Schröer, Preprojective algebras and cluster algebras, preprint (2008) arXiv:math/0804.3168.

[13] D. Hernandez and B. Leclerc, Cluster algebras and quantum affine algebras. arXiv:0903.1452 [math.QA].

[14] R. Kedem, Q-systems as cluster algebras, J. Phys. A: Math. Theor. 41 (2008) 194011 (16 pp). arXiv:0712.2695 [math.RT].

[15] M. Kontsevich, private communication.

[16] A. Kirillov and N. Reshetikhin, Formulas for the multiplicities of the occurrence of irreducible components in the tensor product of representations of simple Lie algebras, Zap. Nauchn. Sem. S.-Peterburg. Otdel. Mat. Inst. Steklov. (POMI) 205 (1993), no. Differentsialnaya Geom. Gruppy Li i Mekh. 13, 30-37, 179.

[17] A. Knutson, T. Tao, C. Woodward, A positive proof of the Littlewood-Richardson rule using the octahedron recurrence. Electron. J. Combin. 11 (2004), Research Paper 61.

[18] A. Kuniba, A. Nakanishi and J. Suzuki, Functional relations in solvable lattice models. I. Functional relations and representation theory. International J. Modern Phys. A 9 no. 30, pp 5215-5266 (1994).

[19] I. Krichever, O. Lipan, P. Wiegmann and A. Zabrodin, Quantum Integrable Systems and Elliptic Solutions of Classical Discrete Nonlinear Equations, Comm. Math. Phys. 188 (1997) 267-304. arXiv:hep-th/9604080.

[20] H. Nakajima, t-analogs of q-characters of Kirillov-Reshetikhin modules of quantum affine algebras, Represent. Theory 7 (2003), 259-274 (electronic).

[21] H. Nakajima, Quiver varieties and cluster algebras. arXiv:0905.0002 [math.QA].

[22] P. Sherman and A. Zelevinsky, Positivity and canonical bases in rank 2 cluster algebras of finite and affine types, Mosc. Math. J. 4 (2004) 947-974. arXiv:math/0307082 [math.RT].

[23] D. Speyer, Perfect matchings and the octahedron recurrence, J. Algebraic Comb. 25 No 3 (2007) 309-348. arXiv:math/0402452 [math.CO].

[24] A. Zelevinsky, Quantum cluster algebras. Oberwolfach talk, February 2005 tt arXiv:0502260 [math.QA]. 